\title{
Effects of capsule on surface diffuse reflectance spectroscopy of the subcapsular parenchyma of a solid organ
}

Daqing Piao

Halen Borron

Alan Hawxby

Harlan Wright

Erin M. Rubin 


\title{
Effects of capsule on surface diffuse reflectance spectroscopy of the subcapsular parenchyma of a solid organ
}

\author{
Daqing Piao, ${ }^{a, b, \star}$ Halen Borron, ${ }^{c}$ Alan Hawxby, ${ }^{d}$ Harlan Wright, ${ }^{d}$ and Erin M. Rubin ${ }^{e}$ \\ ${ }^{a}$ Oklahoma State University, School of Electrical and Computer Engineering, Stillwater, Oklahoma, United States \\ bOklahoma State University, Department of Veterinary Clinical Sciences, Center for Veterinary Health Sciences, Stillwater, \\ Oklahoma, United States \\ 'University of Oklahoma Health Sciences Center, College of Medicine, Oklahoma City, Oklahoma, United States \\ dUniversity of Oklahoma Health Sciences Center, Oklahoma Transplant Center, Oklahoma City, Oklahoma, United States \\ eUniversity of Oklahoma Health Sciences Center, Department of Pathology, Oklahoma City, Oklahoma, United States
}

\begin{abstract}
We hypothesize that the capsular optical properties and thickness combined affect how accurate the diffuse reflectance on the surface of a capsular solid organ represents that on the subcapsular parenchyma. Monte Carlo simulations on two-layer geometries evaluated how a thin superficial layer with the thickness from 10 to $1000 \mu \mathrm{m}$ affected the surface diffuse reflectance over a source-detector separation spanning 0.01 to $10 \mathrm{~mm}$. The simulations represented the superficial layer presenting various contrasts concerning refractive index, anisotropy factor, absorption coefficient, and reduced scattering coefficient, versus those of the subsurface main medium. An analytical approach modeled the effects of the superficial layer of various thicknesses and optical properties on diffuse reflectance. Diffuse reflectance spectroscopy was performed ex vivo on 10 fresh human livers and 9 fresh human kidneys using a surface probe with a 3-mm source-detector separation. The difference of the device-specific diffuse reflectance on the organ between with the capsule and without the capsule has significantly greater spectral variation in the kidney than in the liver. The significantly greater spectral deviation of surface diffuse reflectance between with and without the capsule in the kidney than in the liver was analytically accountable by considering the much thicker capsule of the kidney than of the liver. (๑) 2018 Society of Photo-Optical Instrumentation Engineers (SPIE) [DOI: 10.1117/1.JBO.23.12.121602]
\end{abstract}

Keywords: diffuse reflectance spectroscopy; capsule; light-tissue interaction; diffuse optical spectroscopy; functional near-infrared spectroscopy.

Paper 180052SSR received Jan. 23, 2018; accepted for publication Apr. 16, 2018; published online Jul. 27, 2018.

\section{Introduction}

Represented by the fundamental demonstration in 1977 by Jobsis, ${ }^{1}$ noninvasive spectroscopic assessment of the tissue functionality using diffuse photon in the visible and near-infrared spectra has been applied with various levels of clinical readiness to disease diagnosis, ${ }^{2-4}$ treatment monitoring, ${ }^{5-7}$ and surgery regime. ${ }^{8-11}$ Spectral measurement of the diffuse photon remitted from the object surface, which is referred to as diffuse reflectance spectroscopy (DRS), ${ }^{12}$ diffuse optical spectroscopy (DOS),${ }^{13,14}$ or functional near-infrared spectroscopy, ${ }^{15}$ is among the simplest photon-based techniques suitable for in vivo organsite application ${ }^{13}$ when taking the conveniences offered by fiberbased contact approach for photon illumination and collection. Equally applicable in the noncontact or remote mode for the assessment of spectral constituents of an object, ${ }^{16}$ DRS is also indispensable in the quality assurance of encapsulation of powdered therapeutic compounds ${ }^{17,18}$ and agricultural surveying of some fruits. ${ }^{19,20}$

Diffuse reflectance measurements acquire the spectral remission from the medium surface to assess the spectral alteration caused by tissue spectral heterogeneities within the volume

*Address all correspondence to: Daqing Piao, E-mail: daqing.piao@okstate .edu of sampling. How sensitive DRS is to the tissue spectral heterogeneity is affected by both the spectral contrast of the heterogeneity over the baseline tissue and the distance between the position of photon injection into the medium and the site of light collection from the medium. No tissue is microscopically homogeneous along the depth or the lateral extension sampled by DRS. But the measurement of light diffusely traversed through a volume of tissue allows DRS to sense the lumped optical properties of the tissue. Lacking spatially resolved information, DRS is useful in revealing the composite effect of the spectral alterations caused by the microscopic parenchymal heterogeneities in absorption and/or scattering, which are secondary to changes in biochemical function or pathology. However, when the tissue volume sampled by DRS has a superficial layer that is optically heterogeneous compared with the layer below it, as in the cases of DRS of the subcutaneous tissue, the surface layer that is not the subject of sampling could contaminate the DRS measurement of the medium underlying it.

Continuous-wave (CW) DRS is particularly suitable for application in the intraoperative setting for tissue quality assessment because of its potential for noninvasive evaluation of tissue pathological conditions and real-time responses to tissue biochemical changes. Nilsson et al. ${ }^{21}$ have used CW DRS covering

$1083-3668 / 2018 / \$ 25.00$ @ 2018 SPIE 
a wide spectral range of 450 to $1550 \mathrm{~nm}$ with a source-detector separation (SDS) of $2.5 \mathrm{~mm}$ to evaluate the injury of liver tissue. From the intraoperative and ex vivo measurements on both the surface and cross section of human livers, they have projected that surface DRS measurement of the liver represents the DRS on the cut section of the liver. The DRS on the surface of the liver in the absence of the capsule was shown to be indistinguishable from that on the cross section of the liver over the entire spectral range measured, indicating the spatial uniformity of bulk optical properties of liver parenchyma. However, there were observations suggesting that the DRS of the liver in the presence of the capsule could differ from that of the liver parenchyma. Between the DRS on the surface of the liver in the presence of the capsule and that on the cross section of the liver, the profiles were nearly identical over 1000 to $1550 \mathrm{~nm}$, but a small albeit consistent elevation of the former comparing with the latter could be seen over the 600 - to 1000 -nm range. Between the DRS on the surface of the liver in the presence of the capsule and that on the cross-sectional tumor, however, the deviation was significant over 400 to $1100 \mathrm{~nm}$. These observations argued that the effect of capsule on DRS may not always be negligible when performing on the liver with pathological changes, and when the capsular effect becomes nonnegligible then not accounting for it would cause incorrect reconstruction of the parenchyma chromophore components or scattering parameters based on DRS performed on the surface of the intact liver.

CW DRS has also been tested for the measurement of renal ischemia during laparoscopic partial nephrectomy. ${ }^{22}$ Using a laparoscopic applicator probe with a SDS of $2 \mathrm{~mm}$ and spectral responses over 500- to 650-nm range, the DRS measured on the capsular surface of the kidney was used to estimate the absorption properties of the renal tissue at normoxia ( $82 \%$ oxygen saturation) and ischemia ( $0 \%$ oxygen saturation) using an inverse Monte Carlo (MC) model validated for a homogeneous medium. When compared with the normalized absorption curve expected for the ischemic tissue using the known absorption spectra for deoxygenated human hemoglobin, the absorption coefficient spectra extracted from reflectance of the capsular kidney tissue deviated variously over the 500 to 600 -nm range, with a maximum underestimation of $\sim 30 \%$ at $550 \mathrm{~nm}$. The underestimation of the absorption at $550 \mathrm{~nm}$, which can cause an underestimation of the ischemia, could be attributed to missing of the capsular layer in the tissue modeled by the inverse MC for diffuse reflectance.

The capsule is a thin collagen-rich layer enclosing a solid organ, such as the liver or kidney. The capsule that differs morphologically from the subcapsular parenchyma also differs from the subcapsular parenchyma in optical properties, most pronouncedly the scattering, ${ }^{23}$ that affect the remission of photon after propagating in the respective tissue. A liver's capsule is very thin, $\sim 10 \mu \mathrm{m},{ }^{24}$ but a kidney's capsule is much thicker, $\sim 200 \mu \mathrm{m} .{ }^{25}$ For a liver capsule that is very thin, as conceptually shown in Fig. 1(a), even when it is optically heterogeneous comparing with the main medium, the length of photon path within the capsular layer may be insignificant comparing with the total length of photon path between a source and a detector separated at millimeter dimensions typical to DRS. As a result, one can expect that the DRS performed on the capsular surface of a liver matches closely with the DRS on the subcapsular or cross-sectional parenchyma of a liver. Comparatively, as the kidney capsule is much thicker as schematically shown in Fig. 1(b), when the capsule is also optically heterogeneous comparing

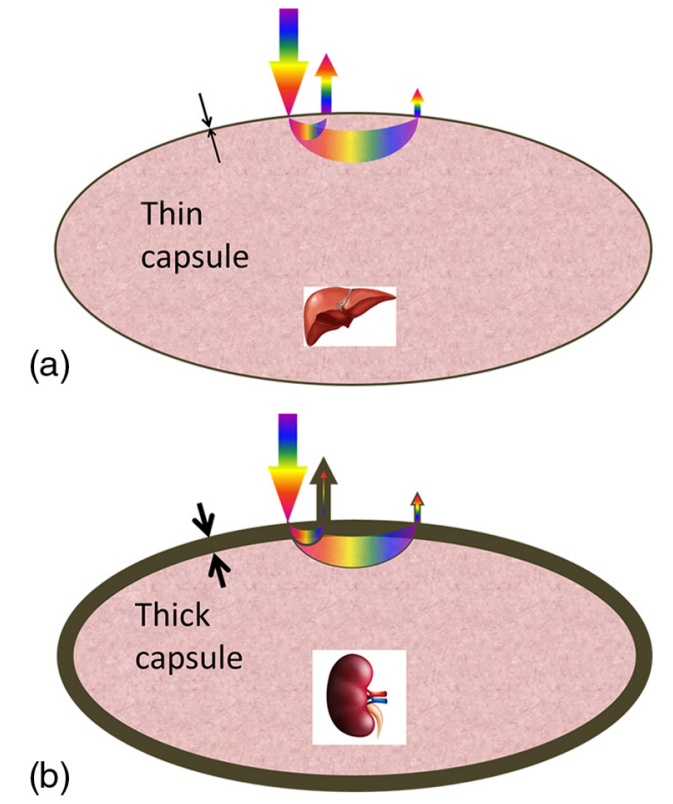

Fig. 1 Schematic illustration of how a thin (A) or thick (B) capsule may affect the surface diffuse reflectance of the subcapsular parenchyma of a capsular organ. If the optical properties of the capsular layer are identical to those of the subcapsular medium, there is no difference of the spatially resolved DRS between with and without the capsule. If the optical properties of the capsular layer pertinent to photon attenuation through scattering and absorption differ from those of the subcapsular medium, the spatially resolved DRS will differ between with and without the capsule. The difference of DRS between with or without the capsule will be more pronounced for a thicker capsule (such as a kidney when compared with a liver) and at a shorter SDS. Surface diffuse reflectance of the spectral properties of the subcapsular parenchyma will be modulated by the spectral properties of the capsule.

with the underlying renal parenchyma, the length of photon path within the capsular layer may be too significant to be neglected from the total length of photon path between a source and a detector separated for DRS. In such cases of the DRS performed on the surface of a parenchyma that is enclosed by a thick capsular layer, extracting the true absorption spectra of the parenchyma using DRS measured on the surface of the intact organ will require the effect of a capsular layer on surface DRS be accounted for.

Accounting for the effect of the capsule on surface DRS fits naturally in the domain of modeling diffuse reflectance from a layered medium. There are numerous modeling studies specific to CW DRS of a layered medium that is commonly simplified as a two-layer planar geometry. ${ }^{26-32} \mathrm{~A}$ recent work targeted DRS of diseases located in the epithelial layer at the tissue surface using probes with a short SDS to sample photons that travel through the epithelial tissue layers. ${ }^{29}$ A two-layer MC lookup table-based inverse model has accurately extracted top layer thickness and scattering when the top layer thickness ranges from 0 to $550 \mu \mathrm{m}$ at 0.37 - and $0.74-\mathrm{mm}$ SDSs and found that the accuracy of top and bottom layer absorption coefficient measurements was highly dependent on top layer thickness. The conclusion of, ${ }^{29}$ which agrees with physical expectation for DRS of a superficial layer that is modified by the properties of the underlying large tissue medium, is useful to assessing how a superficial layer may affect DRS of the underlying large tissue medium at short SDSs, but may not be informative for the many DRS measurements that use SDS in 
the greater-than-millimeter range to probe the main tissue underlying a superficial layer. The majority of other studies apply to diffuse reflectance measurements of subcutaneous tissue that is covered by a relatively thick, millimeter level, superficial layer. The thickness of the superficial layer that these models have addressed has been generally much greater than the thickness of a capsule of a solid organ, such as a liver or a kidney, and the optical properties of the superficial layer investigated for the existing models also represent more of the cutaneous tissue and less of a collagen-rich capsule. As a result, these models may not allow straightforward translation to help evaluate how the spatially resolved diffuse reflectance from a main medium covered by a thin layer may be affected by the very thin superficial layer of capsular-thickness and capsular optical properties.

Among the studies ${ }^{30-32}$ evaluating the diffuse reflectance (including CW and time-domain) in the presence of a superficial layer of millimeter-scale thickness, some have also presented analytical models that are specific to illumination under a uniform collimated wide beam ${ }^{30}$ or having a low or none absorbing medium underlying a superficial layer. ${ }^{26,31}$ An analytical model that will be more robust for quantitating how a thin superficial capsular layer affects surface DRS of the subcapsular main medium is one that applies equally to a low or high absorbing superficial layer or the main medium, a low or high scattering superficial layer or the main medium, at a superficial layer thickness relevant to solid organ capsule, and over a wide-range of SDS whether it is near the point-of-entry or at far-field, concerning the common geometry for a fiber-based DRS.

We hypothesize that the capsular optical properties and thickness combined affect how accurate the DRS performed on the surface of a capsular solid organ represents the DRS on the subcapsular parenchyma. MC simulations were used to evaluate how a thin superficial layer with the thickness ranging from 10 to $1000 \mu \mathrm{m}$ would affect the surface diffuse reflectance for the superficial layer presenting various optical contrasts concerning refractive index, anisotropy factor, absorption coefficient, and reduced scattering coefficient, versus those of the subsurface main planar tissue. An analytical approach has also been developed that models the salient effect of the superficial layer on surface diffuse reflectance as revealed by the MC simulations. The analytical model is applicable to diffuse reflectance at distances ranging from millimeters from the point-ofentry to $10 \mathrm{~s}$ of microns from the point-of-entry and tissue with the absorption coefficient equal in magnitude to the reduced scattering coefficient. DRS measurements have also been performed ex vivo on 10 fresh human livers and 9 fresh human kidneys, for comparing the device-specific differences of the DRS performed on the capsular surface and on the cross-sectional or subcapsular parenchyma of the same organ. Much greater capsular effect to the device-specific DRS is observed from the kidney than the liver. The much greater capsular effect to the DRS of the kidney than of the liver is projected to be associated with the much thicker capsule of the kidney than the liver, according to spectrally resolved implementation of the analytical model under some realistic assumptions of the capsular and main medium properties.

\section{Methods and Materials}

\subsection{Monte Carlo Simulations}

MC simulations were used to evaluate how the optical contrast of a thin $(<1000-\mu$ m thick $)$ superficial layer with respect to the

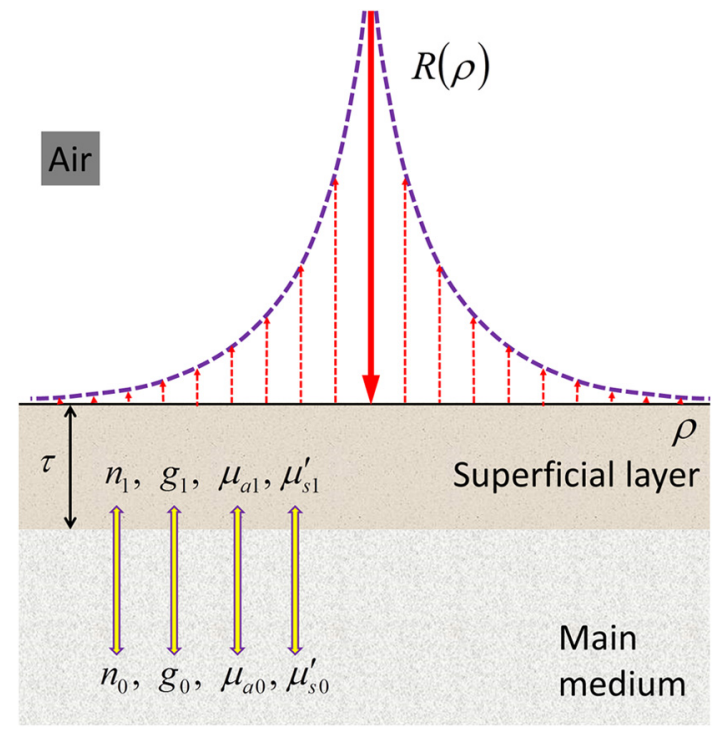

Fig. 2 The two-layer medium geometry in the MC simulation for evaluating the diffuse reflectance $R(\rho)$ at a distance $\rho$ from the position of photon injection to the medium. The superficial layer or the main medium is characterized by four independent optical properties including refractive index $n$, anisotropy factor $g$, absorption coefficient $\mu_{a}$, and reduced scattering coefficient $\mu_{s}^{\prime}$. The optical properties of the main medium are marked with a subscript of "0," and those of the superficial layer "1." The thickness of the superficial layer is $\tau$.

subsurface main medium would affect the spatially resolved diffuse reflectance measured on the surface of the superficial layer. The medium geometry of the MC simulation is shown in Fig. 2. A planar two-layer medium geometry was implemented for evaluating the diffuse reflectance $R(\rho)$ measured at a lateral distance $\rho$ from the point of light injection into the medium. A set of optical properties including the refractive index $n$, the anisotropy factor $g$, the absorption coefficient $\mu_{a}$, and the reduced scattering coefficient $\mu_{s}^{\prime}$ were assigned independently to the superficial layer and the main medium. These four optical properties were used as the primary parameters for each of the two layers. The parameters associated with the main medium are marked by a subscript of " 0 ," and those with the superficial layer by a subscript of " 1 ."

The MC simulation was performed using the "MC solver panel" of Virtual Photonics General-Purpose ATK 2.2.0 Beta, ${ }^{33}$ by custom defining the positions of the detector and the superficial-layer thickness in the off-line editable Input File and changing the medium optical properties using the online graphical user interface. A total of 1000 detector points were placed on the surface of the superficial layer, corresponding to a distance of 0.01 to $10 \mathrm{~mm}$ from the point of photon injection at an interval of $10 \mu \mathrm{m}$. The $10-\mathrm{mm}$ span of the SDS in the MC simulations covers the 3-mm separation between the source and the detector of the single source-detector pair on the applicator probe used for diffuse reflectance measurements from the liver and kidney as will be detailed in the corresponding section. Each MC simulation was executed with a total of 100,000 photons, for a total tissue thickness of $10 \mathrm{~cm}$. The MC simulations were executed with only the Henyey-Greenstein (HG) phase function as it was the one accessible on the virtual photonics $\mathrm{MC}$ solver.

Each MC simulation corresponded to the superficial layer differing from the main medium in only one of the four primary 
parameters including $n_{1}, g_{1}, \mu_{a 1}$, and $\mu_{s 1}^{\prime}$. Two stages of MC simulations were performed. The first stage of MC simulations evaluated the effect of each of the four primary parameters of the superficial layer at a fixed setting of the main medium parameters on the diffuse reflectance for a superficial layer thickness ranging from 10 to $1000 \mu \mathrm{m}$. The individual patterns revealed by the first-stage MC simulations, which constituted $94.9 \%$ of the total number of simulations, regarding how each of the four capsular contrasts over the main medium affected the diffuse reflectance were used to gauge the analytical model development. As will be shown from the first-stage of the MC simulations conducted for a fixed setting of the main medium properties, the diffuse reflectance from the two-layer geometry with a thin superficial layer $(\leq 1000-\mu \mathrm{m}$ thick) is not sensitive to $n_{1}$ and $g_{1}$ (or equivalently the contrast of each over its main medium counterpart) over their respective ranges covering more than what a biological tissue will present. These outcomes were based upon to constrain the parameter setting in the next stage of MC simulation that was intended for evaluating the effect of the change of main medium properties on diffuse reflectance in a more pathologically relevant parameter setting of the superficial layer. The subsequent stage of MC simulations thus was performed at the absence of a superficial layer contrast on the refractive index or anisotropy factor over the main medium. In consideration of the high scattering of organ capsule due to collagen content ${ }^{25}$ and a liver capsule being $\sim 10-\mu$ m thick and a kidney capsule being $\sim 200-\mu \mathrm{m}$ thick, the superficial layer in the second stage of MC simulations was thus fixed at a high scattering property with the thicknesses of 10 and $200 \mu \mathrm{m}$ only, whereas the $\mu_{a 0}$ and $\mu_{s 0}^{\prime}$ of the main medium were changed to evaluate how much the main medium property would affect the spatially resolved surface diffuse reflectance at a fixed setting of a highly scattering superficial layer.

Table 1 shows the parameter configuration of the first-stage MC simulations. In these MC simulations, the main medium was kept fixed at the following baseline set of parameters: $n_{0}=1.40, g_{0}=0.90, \mu_{a 0}=0.01 \mathrm{~mm}^{-1}$, and $\mu_{s 0}^{\prime}=1.0 \mathrm{~mm}^{-1}$. To evaluate the effect of the refractive index of the superficial layer to the diffuse reflectance, the $n_{1}$ was changed from 1.30 to 1.50 at a step increment of 0.01 , whereas keeping other parameters constant as $g_{1}=0.90, \mu_{a 1}=0.01 \mathrm{~mm}^{-1}$, and $\mu_{s 1}^{\prime}=$ $1.0 \mathrm{~mm}^{-1}$. To evaluate the effect of the anisotropy factor of the superficial layer to the diffuse reflectance, the $g_{1}$ was changed from 0.80 to 0.99 at a step increment of 0.01 , whereas keeping other parameters constant as $n_{1}=1.40, \mu_{a 1}=$ $0.01 \mathrm{~mm}^{-1}$, and $\mu_{s 1}^{\prime}=1.0 \mathrm{~mm}^{-1}$. To evaluate the effect of the absorption properties of the superficial layer to the diffuse reflectance, the $\mu_{a 1}$ was changed from 0.001 to $0.01 \mathrm{~mm}^{-1}$ at a step increment of $0.001 \mathrm{~mm}^{-1}$, then from 0.01 to $0.1 \mathrm{~mm}^{-1}$ at a step increment of $0.01 \mathrm{~mm}^{-1}$, and thereafter from 0.1 to $1.0 \mathrm{~mm}^{-1}$ at a step increment of $0.1 \mathrm{~mm}^{-1}$, whereas keeping other parameters constant as $n_{1}=1.40, g_{1}=0.90$, and $\mu_{s 1}^{\prime}=$ $1.0 \mathrm{~mm}^{-1}$. To evaluate the effect of the reduced scattering properties of the superficial layer to the diffuse reflectance, the $\mu_{s 1}^{\prime}$ was changed from 0.1 to $1.0 \mathrm{~mm}^{-1}$ at a step increment of $0.1 \mathrm{~mm}^{-1}$, and then from 1.0 to $10.0 \mathrm{~mm}^{-1}$ at a step increment of $1.0 \mathrm{~mm}^{-1}$, whereas keeping other parameters constant as $n_{1}=1.40, g_{1}=0.90$, and $\mu_{a 1}=0.01 \mathrm{~mm}^{-1}$. The diffuse reflectance at any of the aforementioned setting of the optical parameters of the superficial layer was evaluated at the superficial layer thickness ranging from 10 to $100 \mu \mathrm{m}$ at a step increment of $10 \mu \mathrm{m}$, and then from 100 to $1000 \mu \mathrm{m}$ at a step increment of $100 \mu \mathrm{m}$. These configurations resulted in $\sim 1670$ MC simulations corresponding to the superficial layer that differs from the main medium in one of four primary optical properties, not including the duplicated baseline sets.

Table 2 shows the parameter configuration of the secondstage MC simulations specific to superficial layer with fixed optical properties including a highly reduced scattering coefficient and an underlying main medium at various absorptions and reduced scattering coefficients. In these MC simulations, the properties of the superficial layer were kept fixed at the following parameters: $n_{1}=1.40, g_{1}=0.90, \mu_{a 1}=0.01 \mathrm{~mm}^{-1}$, and $\mu_{s 1}^{\prime}=10.0 \mathrm{~mm}^{-1}$. To evaluate how the absorption of the main medium affects the diffuse reflectance, the $\mu_{a 0}$ was changed from 0.001 to $0.01 \mathrm{~mm}^{-1}$ at a step increment of $0.001 \mathrm{~mm}^{-1}$, then from 0.01 to $0.1 \mathrm{~mm}^{-1}$ at a step increment of $0.01 \mathrm{~mm}^{-1}$, and thereafter from 0.1 to $1.0 \mathrm{~mm}^{-1}$ at a step increment of $0.1 \mathrm{~mm}^{-1}$, with the other parameters of the main medium kept constant as $n_{0}=1.40, g_{0}=0.90$, and $\mu_{s 0}^{\prime}=1.0 \mathrm{~mm}^{-1}$. To evaluate how the reduced scattering of the main medium affects the diffuse reflectance, the $\mu_{s 0}^{\prime}$ was changed from 0.1 to $1.0 \mathrm{~mm}^{-1}$ at a step increment of $0.1 \mathrm{~mm}^{-1}$, and then from 1.0 to $10.0 \mathrm{~mm}^{-1}$ at a step increment of $1.0 \mathrm{~mm}^{-1}$, with the other parameters of the main medium kept constant as $n_{0}=$ $1.40, g_{0}=0.90$, and $\mu_{a 0}=0.01 \mathrm{~mm}^{-1}$. The range of the absorption coefficient of the main medium spanning three decades from 0.001 to $1.0 \mathrm{~mm}^{-1}$ and the range of the reduced scattering coefficient of the main medium spanning two decades from 0.1 to $10.0 \mathrm{~mm}^{-1}$ cover the optical properties typical to soft biological tissues including the liver and kidney. ${ }^{34}$ The diffuse reflectance at any of the aforementioned setting of the optical parameters of the main medium was evaluated at the superficial layer thicknesses of 10 and $200 \mu \mathrm{m}$ only as these two numbers represent, respectively, the thickness of the liver capsule and kidney capsule. These configurations resulted in over $90 \mathrm{MC}$ simulations corresponding to the main medium that differs from the superficial layer in one of the four primary optical properties. Tables 1 and 2 when combined amount to $\sim 1760$ MC simulations corresponding to the semi-infinite tissue geometry containing a superficial layer that is optically heterogeneous comparing with the underlying main medium. These MC simulations were executed at random sequences on multiple computers having various system configurations.

\subsection{Analytical Model of Surface Diffuse Reflectance From a Semi-Infinite Medium in the Presence of a Thin Superficial Layer}

The effect of capsule on the surface diffuse reflectance can be appreciated by identifying how the surface diffuse reflectance of the subcapsular parenchyma at the presence of the capsule may differ from that at the absence of the capsule. At the absence of the capsule, the parenchyma, which is referred to as the main or baseline medium, approximates a homogeneous domain of photon propagation for surface diffuse reflectance. The presence of the capsule introduces a thin superficial layer that may also be optically heterogeneous in comparison with the main medium. How much this capsular layer changes the surface diffuse reflectance from the subsurface main medium when compared with the baseline will be affected by not only the extent of the contrasts of the optical properties of the capsular layer over the main medium but also the thickness of the capsule. Apparently, if the superficial layer presents no contrasts of the optical properties over the main medium, the presence of the superficial layer shall 
Piao et al.: Effects of capsule on surface diffuse reflectance spectroscopy of the subcapsular...

Table 1 The properties of the superficial layer at fixed main medium properties.

$R(\rho)\left(\mathrm{mm}^{-2}\right)$ diffuse reflectance at an SDS of $\rho(\mathrm{mm}), \rho=[0.01,10](\mathrm{mm})$ at a step size of $10 \mu \mathrm{m}$

\begin{tabular}{|c|c|c|c|c|}
\hline & $\begin{array}{l}\text { How does } n_{1} \\
\text { affect } R(\rho)\end{array}$ & $\begin{array}{l}\text { How does } g_{1} \\
\text { affect } R(\rho)\end{array}$ & $\begin{array}{l}\text { How does } \mu_{\mathrm{a} 1} \\
\text { affect } R(\rho)\end{array}$ & $\begin{array}{l}\text { How does } \mu_{s 1}^{\prime} \\
\text { affect } R(\rho)\end{array}$ \\
\hline$\tau=[10,1000](\mu \mathrm{m})$ & $n_{1}=[1.30,1.50]$ & $g_{1}=[0.80,0.99]$ & $\mu_{\mathrm{a} 1}=[0.001,1]\left(\mathrm{mm}^{-1}\right)$ & $\mu_{s 1}^{\prime}=[0.1,10]\left(\mathrm{mm}^{-1}\right)$ \\
\hline$<<10>>$ & 1.30 & 0.80 & 0.001 & 0.1 \\
\hline 20 & 1.31 & 0.81 & 0.002 & 0.2 \\
\hline 30 & 1.32 & 0.82 & 0.003 & 0.3 \\
\hline 40 & 1.33 & 0.83 & 0.004 & 0.4 \\
\hline 50 & 1.34 & 0.84 & 0.005 & 0.5 \\
\hline 60 & 1.35 & 0.85 & 0.006 & 0.6 \\
\hline 70 & 1.36 & 0.86 & 0.007 & 0.7 \\
\hline 80 & 1.37 & 0.87 & 0.008 & 0.8 \\
\hline 90 & 1.38 & 0.88 & 0.009 & 0.9 \\
\hline 100 & 1.39 & 0.89 & $<0.01>$ & $<1.0>$ \\
\hline$<<200>>$ & $<1.40>$ & $<0.90>$ & 0.02 & 2.0 \\
\hline 300 & 1.41 & 0.91 & 0.03 & 3.0 \\
\hline 400 & 1.42 & 0.92 & 0.04 & 4.0 \\
\hline 500 & 1.43 & 0.93 & 0.05 & 5.0 \\
\hline 600 & 1.44 & 0.94 & 0.06 & 6.0 \\
\hline 700 & 1.45 & 0.95 & 0.07 & 7.0 \\
\hline 800 & 1.46 & 0.96 & 0.08 & 8.0 \\
\hline 900 & 1.47 & 0.97 & 0.09 & 9.0 \\
\hline \multirow[t]{2}{*}{10,000} & 1.48 & 0.98 & 0.1 & 10.0 \\
\hline & 1.49 & 0.99 & 0.2 & \\
\hline \multirow{8}{*}{$\begin{array}{l}\text { At each } \tau \text {, the dependence } \\
\text { of } R(\rho) \text { on one parameter } \\
\text { of the superficial layer } \\
\text { is evaluated }\end{array}$} & 1.50 & & 0.3 & \\
\hline & & & 0.4 & \\
\hline & & & 0.5 & \\
\hline & & & 0.6 & \\
\hline & & & 0.7 & \\
\hline & & & 0.8 & \\
\hline & & & 0.9 & \\
\hline & & & 1.0 & \\
\hline main medium thickness $=10 \mathrm{~cm}$ & $n=1.40$ & $g=0.90$ & $\mu_{a}=0.01\left(\mathrm{~mm}^{-1}\right)$ & $\mu_{s}^{\prime}=1.0\left(\mathrm{~mm}^{-1}\right)$ \\
\hline \# of simulations & $19 \times 21=399$ & $19 \times 20=380$ & $19 \times 28=532$ & $19 \times 19=361$ \\
\hline
\end{tabular}

$<X>$ indicates a default value of the parameter at baseline. $\langle\langle X\rangle>$ indicates the layer thickness representing that of the liver or kidney capsules. 
Piao et al.: Effects of capsule on surface diffuse reflectance spectroscopy of the subcapsular...

Table 2 The properties of the main medium at fixed superficial layer properties.

$R(\rho)\left(\mathrm{mm}^{-2}\right)$ diffuse reflectance at an SDS of $\rho(\mathrm{mm}), \rho=[0.01,10](\mathrm{mm})$ at a step size of $10 \mu \mathrm{m}$

\begin{tabular}{|c|c|c|c|c|}
\hline & & & $\begin{array}{c}R(\rho) \text { dependence } \\
\text { on } \mu_{a 0} \text { (the absorption } \\
\text { coefficient of the } \\
\text { main medium) }\end{array}$ & $\begin{array}{l}R(\rho) \text { dependence } \\
\text { on } \mu_{s 0}^{\prime} \text { (the reduced } \\
\text { scattering coefficient } \\
\text { of the main medium) }\end{array}$ \\
\hline$\tau=10,200(\mu \mathrm{m})$ & $n_{1}=1.40$ & $g_{1}=0.90$ & $\mu_{a 1}=0.01\left(\mathrm{~mm}^{-1}\right)$ & $\mu_{s 1}^{\prime}=10\left(\mathrm{~mm}^{-1}\right)$ \\
\hline Main medium & $n_{0}=1.40$ & $g_{0}=0.90$ & $\mu_{a 0}\left(\mathrm{~mm}^{-1}\right)$ & $\mu_{s 0}^{\prime}\left(\mathrm{mm}^{-1}\right)$ \\
\hline \multirow{28}{*}{$\begin{array}{l}\text { At each } \tau \text {, the dependence of } \\
R(\rho) \text { on one parameter of } \\
\text { the main medium absorption } \\
\text { or reduced scattering is evaluated }\end{array}$} & & & 0.001 & 0.1 \\
\hline & & & 0.002 & 0.2 \\
\hline & & & 0.003 & 0.3 \\
\hline & & & 0.004 & 0.4 \\
\hline & & & 0.005 & 0.5 \\
\hline & & & 0.006 & 0.6 \\
\hline & & & 0.007 & 0.7 \\
\hline & & & 0.008 & 0.8 \\
\hline & & & 0.009 & 0.9 \\
\hline & & & $<0.01>$ & $<1.0>$ \\
\hline & & & 0.02 & 2.0 \\
\hline & & & 0.03 & 3.0 \\
\hline & & & 0.04 & 4.0 \\
\hline & & & 0.05 & 5.0 \\
\hline & & & 0.06 & 6.0 \\
\hline & & & 0.07 & 7.0 \\
\hline & & & 0.08 & 8.0 \\
\hline & & & 0.09 & 9.0 \\
\hline & & & 0.1 & 10.0 \\
\hline & & & 0.2 & \\
\hline & & & 0.3 & \\
\hline & & & 0.4 & \\
\hline & & & 0.5 & \\
\hline & & & 0.6 & \\
\hline & & & 0.7 & \\
\hline & & & 0.8 & \\
\hline & & & 0.9 & \\
\hline & & & 1.0 & \\
\hline \# of simulations & & & $2 \times 28=56$ & $2 \times 19=38$ \\
\hline
\end{tabular}


have no effect to the surface diffuse reflectance of the composite media containing the superficial layer and the main medium, regardless of the thickness of the capsular layer. In contrast, if the capsular layer is optically heterogeneous comparing with the main medium, the effect of this capsular layer on the surface diffuse reflectance will decrease and eventually vanish as the capsular layer becomes thinner and disappears. An analytical model addressing the effect of the superficial layer on the surface diffuse reflectance from a layered medium thus shall converge to the case of surface diffuse reflectance from a homogeneous medium when either the contrast of the optical properties of the superficial layer over the main medium vanishes or the thickness of the superficial layer zeros. Conversely, the effect of the superficial layer to the surface diffuse reflectance of the multilayer medium may be accounted for by a differential change of the surface diffuse reflectance from a baseline homogeneous medium, which shall elevate as the contrast of the optical properties of the superficial layer over the main medium increases or the superficial layer thickens. These considerations, heretofore, substantiate the analytical model development as detailed in the following.

Recently, we have demonstrated a simple, convenient, and transparent model of diffuse reflectance from a homogeneous semi-infinite medium ${ }^{33}$ that has been validated over an SDS ranging from 0.01 to $10 \mathrm{~mm}$ and tissue diffusing conditions including the absorption coefficient as strong in magnitude as the scattering coefficient $\left(\mu_{a} \sim \mu_{s}^{\prime}\right)$ at a medium reduced scattering coefficient of $\mu_{s}^{\prime}=1.0 \mathrm{~mm}^{-1}$. The central scheme of this model is a master-slave dual-source configuration that sets two virtual sources, with one being the primary or the master source and the other being the secondary or the slave source, whose position and strength are dependent upon the master source. Both the master source and the slave source are placed along the ballistic line of photon injection for the apparent geometric symmetry, as shown in Fig. 3(a). For a homogeneous medium confined in a semi-infinite geometry that is illuminated by a directional point-beam, the master source is set at a depth of one reduced scattering pathlength or $1 / \mu_{s}^{\prime}$, and the slave source is set to be much closer to the medium boundary at a depth less than one scattering step-size [i.e., the depth $<1 / \mu_{s}$ or $(1-g) / \mu_{s}^{\prime}$ ]. The master source is similar, albeit not identical, to the commonly implemented isotropic source in delivering the far-field pattern of surface photon irradiation for diffuse reflectance. The slave source that is set to be much closer to the medium boundary than the master source is conveniently produced the aggressive peaking of the diffuse reflectance toward the point-of-entry as applying to the common fiberbased contact irradiation geometry involving a point-injection of photons into the medium.

We modify this master-slave dual-source approach to model the spatially resolved diffuse reflectance from an otherwise homogeneous semi-infinite medium in the presence of a thin superficial layer, as shown in Fig. 3(b). The modification introduces a differential change to the master-slave dual-source configuration to account for the change to surface diffuse reflectance from the composite media due to the presence of the thin superficial layer when compared with that from the homogeneous main medium only-referred to hereafter as the baseline. When either the contrast of the optical properties of the superficial layer with a fixed thickness over the main medium or the thickness of the superficial layer with a fixed contrast of the optical properties over the main medium increases, the change to the diffuse reflectance with respect to the baseline shall increase. The increased change to the diffuse reflectance at a stronger superficial layer as shown in Fig. 3(c) (corresponding to either a greater contrast of the optical properties of the superficial layer over the main medium for a given thickness of the superficial layer or a thicker superficial layer at a given contrast of the optical properties of the superficial layer over the main medium) at the same main medium could cause the master-slave sources to deviate more from their respective baseline settings. As the superficial layer becomes more significant in terms of the contrast of its optical properties over the main medium or the layer thickens, the effective medium properties associated with each source could also vary as to affect the photon irradiation contributed by the individual source. In regard to the changes in the diffuse reflectance from the baseline, a differential source is thus introduced. The contribution to the diffuse reflectance by the differential source is set to increase as the superficial layer becomes stronger in either its contrast of optical properties over the main medium or layer thickness and conversely to disappear as the superficial layer vanishes due to no contrast of optical properties over the main medium or too thin to be accounted for.

The following analytical development treats the diffuse reflectance from a semi-infinite medium containing a thin superficial layer overlaying an otherwise homogeneous main medium as the composition of three photon irradiation components: a master component, a slave component, and a differential component. When the superficial layer becomes stronger due to either increasing of the contrast of optical properties over the main medium or thickening of the layer, the differential component is set to increase, whereas the master and the slave components are set to deviate from their corresponding baseline values. The decomposition of the surface photon irradiation to three components must also warrant that when the superficial layer vanishes at the cases of either the superficial layer presenting no contrast of optical properties over the main medium or the thickness of the superficial layer with an accountable contrast of optical properties over the main medium approaching zero, the differential component must vanish, whereas the master component and the slave component shall return to their respective baseline values associated with a homogeneous main medium.

In implementing the master-slave dual-source configuration for the diffuse reflectance from a semi-infinite homogeneous medium, ${ }^{33}$ the contribution to the surface diffuse photon irradiance by the master source and the slave source was computed using the same set of basic formula, with only the variables differing according to whether it is associated with the master source or the slave source. The basic set of formula for the master-slave dual-source configuration was established according to the image-source approach commonly associated with the implementation of an extrapolated zero-boundary condition ${ }^{35,36}$ that sets zero the photon fluence rate on a virtual boundary slightly away from the physical boundary. The position of the extrapolated boundary on which the photon fluence rate is set at zero is determined by the refractive index mismatch between the two media forming the physical boundary. ${ }^{35,36}$ Similarly in this work of modeling the diffuse reflectance from a semi-infinite composite medium containing a thin superficial layer overlying an otherwise homogeneous medium, the master, slave, and differential components of the surface diffuse photon irradiance are computed also using the same set of basic formula governed by the image-source approach associated with 


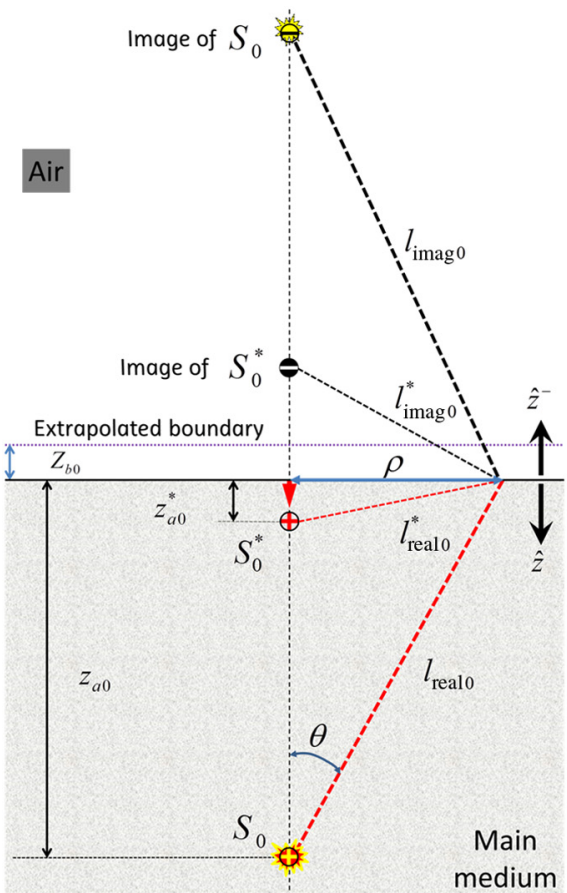

(a)

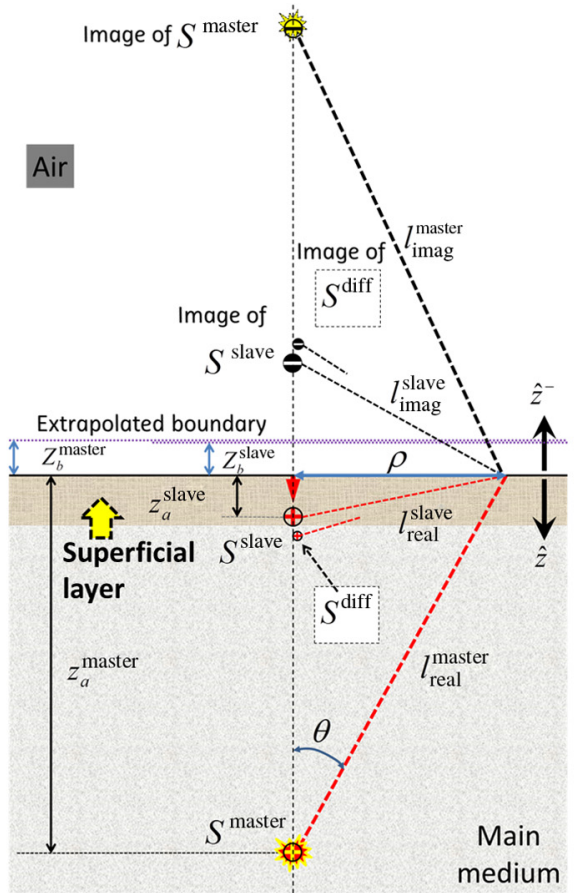

(b)

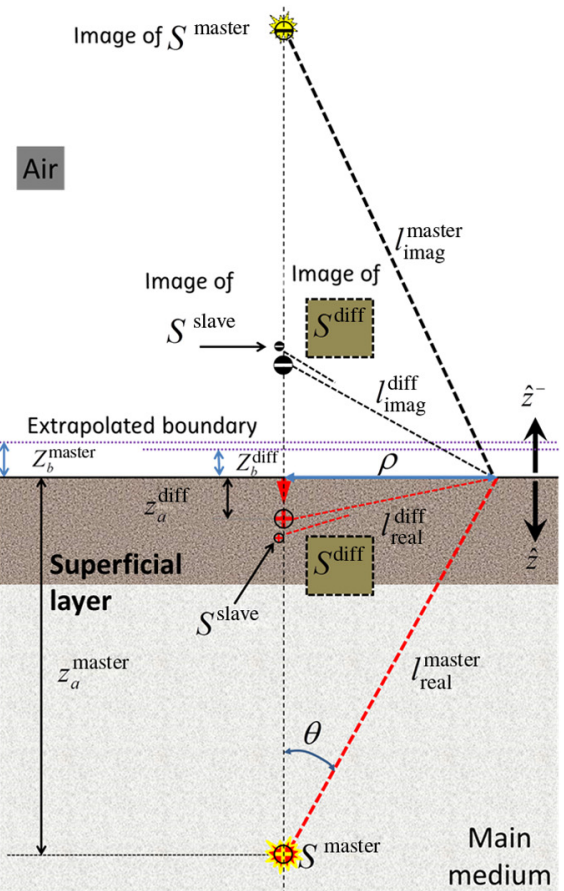

(c)

Fig. 3 The medium geometry used for the analytical model development. (a) The geometry of the medium in the absence of the superficial layer, when only the master source and the slave source are considered. The master-source $S$ locates at a depth of $z_{a}=1 / \mu_{s}^{\prime}$ from the point of entry. The slave-source $S^{*}$ locates at a depth of $z_{a}^{*}$ that is proximal to the point of entry than the position of a depth of $1 / \mu_{s}$. The image source of $S$ is at $z_{a}+2 z_{b}$ away from the medium surface, and the image source of $S^{*}$ is at $z_{a}^{*}+2 z_{b}$ away from the medium surface. (b) The geometry of the medium in the presence of a superficial layer that does not change the surface diffuse reflectance significantly when compared with that in the absence of the superficial layer. The master-source $S^{\text {master }}$ locates at a depth $z_{a}^{\text {master }}$ from the point of entry. The slave-source $S^{\text {slave }}$ locates at a depth of $z_{a}^{\text {slave }}$ that is much proximal to the point of entry than the position of the master source. A differential source $S^{\text {diff }}$ appears with its position and strength dependent upon the significance of the superficial layer in terms of the optical contrast over the main medium and the layer thickness. The image source of $S^{\text {master }}$ is at $z_{a}^{\text {master }}+$ $2 z_{b}^{\text {master }}$ away from the medium surface. The image source of $S^{\text {slave }}$ is at $z_{a}^{\text {slave }}+2 z_{b}^{\text {slave }}$ away from the medium surface. The image source of $S^{\text {diff }}$ is at $z_{a}^{\text {diff }}+2 z_{b}^{\text {diff }}$ away from the medium surface. (c) The geometry of the medium in the presence of a superficial layer that changes the surface diffuse reflectance significantly when compared with that in the absence of the superficial layer. The master-source $S^{\text {master }}$ locates at a depth $z_{a}^{\text {master }}$ from the point of entry. A weak slave-source $S^{\text {slave }}$ locates at a depth of $z_{a}^{\text {slave }}$ that is much proximal to the point of entry than the position of the master source. A strong differential source $S^{\text {diff }}$ appears with its position and strength dependent upon the significance of the superficial layer in terms of the optical contrast over the main medium and the layer thickness. The image source of $S^{\text {master }}$ is at $z_{a}^{\text {master }}+2 z_{b}^{\text {master }}$ away from the medium surface. The image source of $S^{\text {slave }}$ is at $z_{a}^{\text {slave }}+2 z_{b}^{\text {slave }}$ away from the medium surface. The image source of $S^{\text {diff }}$ is at $z_{a}^{\text {diff }}+2 z_{b}^{\text {diff }}$ away from the medium surface.

the extrapolated zero-boundary condition but with the variables differing among the three components, according to the contrast of the optical properties of the superficial layer over the main medium and the thickness of the superficial layer. In the following, the basic set of formula governed by the image-source approach associated with the extrapolated zero-boundary condition and applying equally to the master, slave, and differential components is presented first. The principle of formulating the variables associated, respectively, with the master, slave, and differential components for the variables to depend upon the contrast of the optical properties of the superficial layer over the main medium and the thickness of the superficial layer is described afterward. The actual forms of all variables used for the master, slave, and differential components are listed in Appendix A.
In the analytics that follows, any variable or entity that is associated with the master component is marked by a superscript of "master," the slave component a superscript of "slave," and the differential component a superscript of "diff." To be consistent with the parameter notations used for the MC simulations, any variable that is uniquely defined by the properties of only the main medium is characterized by an ending subscript of " 0 ," and its superficial layer counterpart by an ending subscript of " 1. ." The four parameters including $n, g, \mu_{a}$, and $\mu_{s}^{\prime}$ are considered as the primary parameters as in MC simulations for either the main medium or the superficial layer, inferring that all other parameters used for deriving the diffuse reflectance are secondary parameters to be determined by these four primary parameters. 


\subsubsection{Basic notations of variables and set of formula for the surface diffuse reflectance}

The three geometries shown in Fig. 3 for generalizing the analytical configuration heretofore are used hereafter to assist the analytical model development that accounts for the effect of superficial layer to the surface diffuse reflectance. The three geometries of Fig. 3 are further detailed in the following:

A. Corresponds to the geometry of the medium in the absence of the superficial layer, when only the master source and the slave source need to be considered. A directional point-beam incident at the boundary is represented by the red arrow-head. The master source $S$ locates at a depth of $z_{a}=1 / \mu_{s}^{\prime}$ from the point of entry. The slave-source $S^{*}$ locates at a depth of $z_{a}^{*}$ that is proximal to the point of entry than the position of a depth of $1 / \mu_{s}$. The position of the extrapolated zero-boundary is set at a distance of $z_{b}=2 A D$ away from the physical boundary, where $A=(1+\xi) /(1-\xi), \xi=-1.440 n^{-2}+$ $0.710 n^{-1}+0.668+0.0636 n, n$ is the refractive index of the diffusive medium when bounding with air, ${ }^{36}$ and $D=\left[3\left(\mu_{a}+\mu_{s}^{\prime}\right)\right]^{-1}$ is the diffusion coefficient of the diffusive medium. With respect to this extrapolated zero boundary, the image source of $S$ is at $z_{a}+2 z_{b}$ away from the medium surface, and the image source of $S^{*}$ is at $z_{a}^{*}+2 z_{b}$ away from the medium surface.

B. Corresponds to the geometry of the medium in the presence of a superficial layer of very thin thickness or very low contrast of optical properties over the main medium that does not change the surface diffuse reflectance significantly when compared with that in the absence of the superficial layer. The directional point-beam incident at the boundary is also represented by the red arrow-head. The master source $S^{\text {master }}$ locates at a depth $z_{a}^{\text {master }}$ from the point of entry. The slave source $S^{\text {slave }}$ locates at a depth of $z_{a}^{\text {slave }}$ that is much proximal to the point of entry than the position of the master source. It is noted that the set of $S^{\text {master }}$ and $z_{a}^{\text {master }}$ of (B) differs from the set of $S$ and $z_{a}$ of (A); similarly, the set of $S^{\text {slave }}$ and $z_{a}^{\text {slave }}$ of (B) differs from the set of $S^{*}$ and $z_{a}^{*}$ of (A). When the superficial layer vanishes due to either thinning or disappearing of its contrast of optical properties over the main medium, the set of $S^{\text {master }}$ and $z_{a}^{\text {master }}$ of (B) reaches the set of $S$ and $z_{a}$ of (A); similarly, the set of $S^{\text {slave }}$ and $z_{a}^{\text {slave }}$ of (B) approaches the set of $S^{*}$ and $z_{a}^{*}$ of (A) when the effect of the superficial layer vanishes. A differential source $S^{\text {diff }}$ appears with its position and strength dependent upon the significance of the superficial layer in terms of the contrast of its optical properties over the main medium and the layer thickness. The extrapolated zero-boundary associated with the master source $S^{\text {master }}$ is placed $z_{b}^{\text {master }}$ away from the medium surface, so the image source of $S^{\text {master }}$ is at $z_{a}^{\text {master }}+2 z_{b}^{\text {master }}$ away from the medium surface. The extrapolated zero-boundary associated with the slave source $S^{\text {slave }}$ is placed $z_{b}^{\text {slave }}$ away from the medium surface, so the image source of $S^{\text {slave }}$ is at $z_{a}^{\text {slave }}+2 z_{b}^{\text {slave }}$ away from the medium surface. The extrapolated zero-boundary not shown in the figure that is associated with the differential source $S^{\text {diff }}$ is placed $z_{b}^{\text {diff }}$ away from the medium surface, so the image source of $S^{\mathrm{diff}}$ is at $z_{a}^{\text {diff }}+2 z_{b}^{\text {diff }}$ away from the medium surface. It can be projected that, when the superficial layer vanishes due to either thinning or disappearing of the contrast of its optical properties over the main medium, all of $z_{b}^{\text {master }}$, $z_{b}^{\text {slave }}$, and $z_{b}^{\text {diff }}$ become $z_{b}$, and the contribution to the diffuse reflectance by $S^{\text {diff }}$ shall vanish (the vanishing can be reached by either a vanishing $S^{\text {diff }}$ or an infinitely large effective attenuation coefficient of the medium).

C. Corresponds to the geometry of the medium in the presence of a superficial layer that changes the surface diffuse reflectance much more significantly when compared with the geometry in (B). The much stronger change by the superficial layer to the surface diffuse reflectance in comparison with (B) can be associated with either a much thicker superficial layer or a much stronger contrast of the optical properties of the superficial layer over the main medium. The directional point-beam incident at the boundary is again represented by the red arrow-head. The master source $S^{\text {master }}$ locates at a depth $z_{a}^{\text {master }}$ from the point of entry. A weak slave source $S^{\text {slave }}$ locates at a depth of $z_{a}^{\text {slave }}$ that is much proximal to the point of entry than the position of the master source. A strong differential source $S^{\text {diff }}$ appears with its position and strength dependent upon the significance of the superficial layer in terms of the contrast of its optical properties over the main medium and the layer thickness. The extrapolated zero-boundary associated with the master source $S^{\text {master }}$ is placed $z_{b}^{\text {master }}$ away from the medium surface, so the image source of $S^{\text {master }}$ is at $z_{a}^{\text {master }}+2 z_{b}^{\text {master }}$ away from the medium surface. The extrapolated zero-boundary not shown in the figure that is associated with the slave source $S^{\text {slave }}$ is placed $z_{b}^{\text {slave }}$ away from the medium surface, so the image source of $S^{\text {slave }}$ is at $z_{a}^{\text {slave }}+2 z_{b}^{\text {slave }}$ away from the medium surface. The extrapolated zero-boundary associated with the differential source $S^{\text {diff }}$ is placed $z_{b}^{\text {diff }}$ away from the medium surface, so the image source of $S^{\text {diff }}$ is at $z_{a}^{\text {diff }}+2 z_{b}^{\text {diff }}$ away from the medium surface. It is noted that, as the effect of the superficial layer to the surface diffuse reflectance becomes stronger due to either the thickening of the layer or increasing of the contrast of its optical properties to the main medium, the set of $S^{\text {master }}$ and $z_{a}^{\text {master }}$ of (C) deviates more from the set of $S$ and $z_{a}$ of (A); similarly, the set of $S^{\text {slave }}$ and $z_{a}^{\text {slave }}$ of (C) deviates more from the set of $S^{*}$ and $z_{a}^{*}$ of (A) as the effect of the superficial layer strengthens. It can also be projected that, when the superficial layer thickens or increases in the contrast of its optical properties over the main medium, the contribution to the diffuse reflectance by $S^{\text {diff }}$ increases and may overwhelm the contribution by $S^{\text {slave }}$.

The variables common to the set of formula of the diffuse reflectance are listed as the following with their dimension or unit indicated by $[x]$ :

$D$ is the $[\mathrm{mm}]$ diffusion coefficient, $\mu_{\text {eff }}$ is the $\left[\mathrm{mm}^{-1}\right]$ effective attenuation coefficient, $z_{a}$ is the $[\mathrm{mm}]$ depth of a source from the medium boundary, $z_{b}$ is the $[\mathrm{mm}]$ distance of the extrapolated zero-boundary from the medium boundary, $l_{\text {real }}$ is the $[\mathrm{mm}]$ distance from a source in the medium to the surface detector, $l_{\text {imag }}$ is the $[\mathrm{mm}]$ distance from an image of a source in the medium with respect to its respective extrapolated zeroboundary to the surface detector, $\rho$ is the $[\mathrm{mm}]$ distance of the detector to the position of photon injection on the surface, 
$S$ is the strength of a source, $\Psi$ is the $\left[\mathrm{mm}^{-2}\right]$ steady-state photon fluence rate, defined as

$\Psi(\rho)=\frac{S}{4 \pi D}\left[\frac{\exp \left(-\mu_{\text {eff }} l_{\text {real }}\right)}{l_{\text {real }}}-\frac{\exp \left(-\mu_{\text {eff }} l_{\text {mag }}\right)}{l_{\text {imag }}}\right]$,

$\left.J\right|_{\left(\hat{z}^{-}\right)}$is the $\left[\mathrm{mm}^{-2}\right]$ steady-state photon flux, where $\hat{z}^{-}$represents a unit vector normal to the medium surface and pointing outward, defined as

$$
\begin{aligned}
\left.J\right|_{\left(\hat{z}^{-}\right)}(\rho)= & \frac{S}{4 \pi}\left[\frac{z_{a}\left(\mu_{\text {eff }} l_{\text {real }}+1\right) \exp \left[-\mu_{\text {eff }} l_{\text {real }}\right]}{\left(l_{\text {real }}\right)^{3}}\right. \\
& \left.+\frac{\left(z_{a}+2 z_{b}\right)\left(\mu_{\text {eff }} l_{\text {imag }}+1\right) \exp \left[-\mu_{\text {eff }} l_{\text {imag }}\right]}{\left(l_{\text {imag }}\right)^{3}}\right],
\end{aligned}
$$

and $R$ is the $\left[\mathrm{mm}^{-2}\right]$ steady-state photon irradiance, defined as ${ }^{33}$

$$
R(\rho)=\frac{1}{\sqrt{2}}\left[\frac{1}{4 \pi} \Psi(\rho)+\left.\frac{3}{4 \pi} J\right|_{\left(\hat{z}^{-}\right)}(\rho)\right] .
$$

\subsubsection{Parameters that are determined by the fixed optical properties of only the main medium or the superfi- cial layer}

The refractive index of the air is fixed at $n_{\text {air }}=1.0$. The following parameters are constants for a fixed set of the optical properties of the main medium and the superficial layer. The primary properties are marked by $[0,1]$ to indicate their associations with either the main medium " 0 " or the superficial layer " 1 ," as listed in the following:

$n_{[0,1]}$ is the refractive index,

$g_{[0,1]}$ is the anisotropy factor,

$\mu_{a[0,1]}$ is the $\left[\mathrm{mm}^{-1}\right]$ absorption coefficient, and

$\mu_{s[0,1]}^{\prime}$ is the $\left[\mathrm{mm}^{-1}\right]$ reduced scattering coefficient.

The secondary properties are also marked by $[0,1]$ to indicate their association with the properties of only the main medium " 0 " or the superficial layer " 1 ," as listed in the following:

Diffusion coefficient $[\mathrm{mm}]$ is defined as

$$
D_{[0,1]}=\frac{1}{3\left(\mu_{a[0,1]}+\mu_{s[0,1]}^{\prime}\right)} .
$$

Effective attenuation coefficient $\left[\mathrm{mm}^{-1}\right]$ is defined as

$\mu_{\mathrm{eff}[0,1]}=\sqrt{\frac{\mu_{a[0,1]}}{D_{[0,1]}}}$

Scattering coefficient $\left[\mathrm{mm}^{-1}\right]$ is defined as

$\mu_{s[0,1]}=\frac{\mu_{s[0,1]}^{\prime}}{\left(1-g_{[0,1]}\right)}$.

A coefficient determined by the refractive index of a medium $^{36}$ is defined as

$$
\begin{aligned}
\xi_{[0,1]}= & -1.440\left(n_{[0,1]}\right)^{-2}+0.710\left(n_{[0,1]}\right)^{-1}+0.668 \\
& +0.0636\left(n_{[0,1]}\right)
\end{aligned}
$$

A coefficient related to the refractive index mismatch between the medium and the air is defined as

$A_{[0,1]}=\frac{1+\xi_{[0,1]}}{1-\xi_{[0,1]}}$.

Slave source index ${ }^{33}$ is defined as

$\eta_{[0,1]}=\left[g_{[0,1]} \cdot \exp \left(1-g_{[0,1]}\right)\right]^{1 / 10}$.

The depth of the master source from the medium boundary ${ }^{33}$ $[\mathrm{mm}]$ is defined as

$z_{a[0,1]}=\frac{1}{\mu_{s[0,1]}^{\prime}}$.

The depth of the slave source from the medium boundary ${ }^{33}$ $[\mathrm{mm}]$ is defined as

$z_{a[0,1]}^{*}=\left[\frac{1}{\mu_{s[0,1]}}\right]^{2} \cdot \mu_{s[0,1]}^{\prime} \cdot\left(1-\eta_{[0,1]}\right)$.

The distance of the extrapolated zero-boundary from the medium-air interface $[\mathrm{mm}]$ is defined as

$z_{b[0,1]}=2 \cdot A_{[0,1]} \cdot D_{[0,1]}$.

The distance of the master source to the detector [mm] is defined as

$l_{\text {real }[0,1]}=\sqrt{\rho^{2}+\left(z_{a[0,1]}\right)^{2}}$.

The distance of the slave source to the detector $[\mathrm{mm}]$ is defined as

$l_{\mathrm{imag}[0,1]}=\sqrt{\rho^{2}+\left(z_{a[0,1]}+2 \cdot z_{b[0,1]}\right)^{2}}$.

The strength of the master source ${ }^{33}$ is defined as

$S_{[0,1]}=1$.

The strength of the slave source with respect to that of the master source ${ }^{33}$ is defined as

$S_{[0,1]}^{*}=\eta_{[0,1]} \cdot \exp \left[-\mu_{\operatorname{eff}[0,1]} \frac{z_{a[0,1]}+z_{a[0,1]}^{*}}{2}\right]$.

\subsubsection{Parameters that are determined by the contrast of absorption or reduced scattering properties between the superficial layer and the main medium}

The following parameters are determined by the contrast of the absorption coefficient or the reduced scattering coefficient between the superficial layer and the main medium.

The index of contrast in absorption between the superficial layer and the main medium is defined as 
$\Delta_{a}=\left(\frac{\mu_{a 1}-\mu_{a 0}}{\mu_{a 1}+\mu_{a 0}}\right)^{2}$

The index of contrast in reduced scattering between the superficial layer and the main medium is defined as

$\Delta_{s^{\prime}}=\left(\frac{\mu_{s 1}^{\prime}-\mu_{s 0}^{\prime}}{\mu_{s 1}^{\prime}+\mu_{s 0}^{\prime}}\right)^{2}$

We introduce a general scaling factor that controls the dependence of the contribution to the surface photon irradiance by the master, slave, or differential sources. The scaling factor when applying to the effective optical properties of the medium takes the following form:

$\varepsilon_{\mathrm{eff}}=1-\left[1-\left(1-\Delta_{a}\right)^{\alpha} \cdot\left(1-\Delta_{s^{\prime}}\right)^{\beta}\right] \cdot \delta^{\gamma}$,

where $\delta=\tau / z_{\text {caps }}$ and $z_{\text {caps }}[\mathrm{mm}]$ is an arbitrary number with the dimension of length and is chosen as $1 \mathrm{~mm}$ in this work, the numerical value $\alpha$ determines the dependence of a respective entity on the contrast in absorption coefficient of the superficial layer over the main medium, the numerical value $\beta$ on the contrast in reduced scattering coefficient of the superficial layer over the main medium, and the numerical value $\gamma$ on the thickness of the superficial layer. A set of numerical values $[\alpha, \beta, \gamma]$ may differ among the master, slave, and differential components of the photon irradiation. Similarly, the scaling factor when applying to the source strength takes the following form:

$\varepsilon_{\text {strength }}=1-\left[1-\left(1-\Delta_{a}\right)^{x} \cdot\left(1-\Delta_{s^{\prime}}\right)^{y}\right] \cdot \delta^{z}$,

where the set of numerical values $[x, y, z]$ that may also differ among the master, slave, and differential components of the photon irradiation determines the dependence of the respective entity on the contrasts in absorption and reduced scattering coefficients of the superficial layer over the main medium and the thickness of the superficial layer. Whichever the set of numerical values is for Eqs. (19) or (20), the scaling factor becomes 1 as either the contrast of the optical properties of the superficial layer over the main medium vanishes or the thickness of the superficial layer zeros, and approaches 0 as either the index of contrast in absorption or the index of contrast in reduced scattering becomes 1. Subtracting the scaling factor of Eqs. (19) or (20) from 1 generates another scaling factor that becomes 0 as either the contrast of the optical properties of the superficial layer over the main medium vanishes or the thickness of the superficial layer zeros, and approaches 1 as either the index of contrast in absorption or the index of contrast in reduced scattering becomes 1 . For some variables, a slightly modified form of the scaling factor is adopted to address the pattern of change of the photon irradiation when the superficial layer is less absorptive or less scattering than the main medium in comparison with when the superficial layer is more absorptive or more scattering than the main medium. The scaling factor in any of those slightly modified forms still conforms to 1 as either the contrast of the optical properties of the superficial layer vanishes or the thickness of the superficial layer zeros, and reaches 0 as either the index of absorption contrast or the index of reduced scattering contrast becomes 1 . The parameters specific to the master, slave, and differential contributions to the surface diffuse reflectance are determined by the contrast of optical properties of the superficial layer over the main medium and the layer thickness, as detailed in Appendix A.

\subsection{Diffuse Reflectance Spectroscopy of Liver and Kidney Using a Lab-on-a-Crate Device}

DRS of liver and kidney specimens was acquired using a device shown in Fig. 4. The DRS device was developed onto a wheeled crate for use in a clinical pathology laboratory. The output of a Halogen light source (Cuda I-150, Jacksonville, Florida) through a fiber-optical light-conduit was coupled by a $40 \times$ achromatic objective lens (Olympus RMS40X, Thorlabs Inc, Newton, New Jersey) to a $400-\mu \mathrm{m}$ fiber that was directed to a custom applicator probe as the source channel for tissue illumination. The light diffusely propagated through the tissue was collected by a second $400-\mu \mathrm{m}$ fiber on the same applicator probe and at a 3-mm distance from the source fiber. The detector fiber was coupled to a compact visible/near-infrared spectrometer (NT58-303; Edmund Optics Inc., Barrington, New Jersey) with a 16-bit data resolution (0-65535).

The applicator probe consisted of a $12.5-\mathrm{mm}$ diameter inner probe and a 2-in.-diameter outer adaptor, both made of black plastic materials. The inner probe contained two $400-\mu \mathrm{m}$ fibers separated at $3 \mathrm{~mm}$ as the source-detector pair for DRS. The $12.5-\mathrm{mm}$ diameter inner probe was tightly plugged into the outer adaptor with an outer diameter of 2 in. $(50.4 \mathrm{~mm})$. Once plugged in, the inner probe and the outer adaptor formed an integral handheld probe of 2 in. in diameter. The outer adaptor of 2 in. in diameter served for two purposes: (1) to suppress

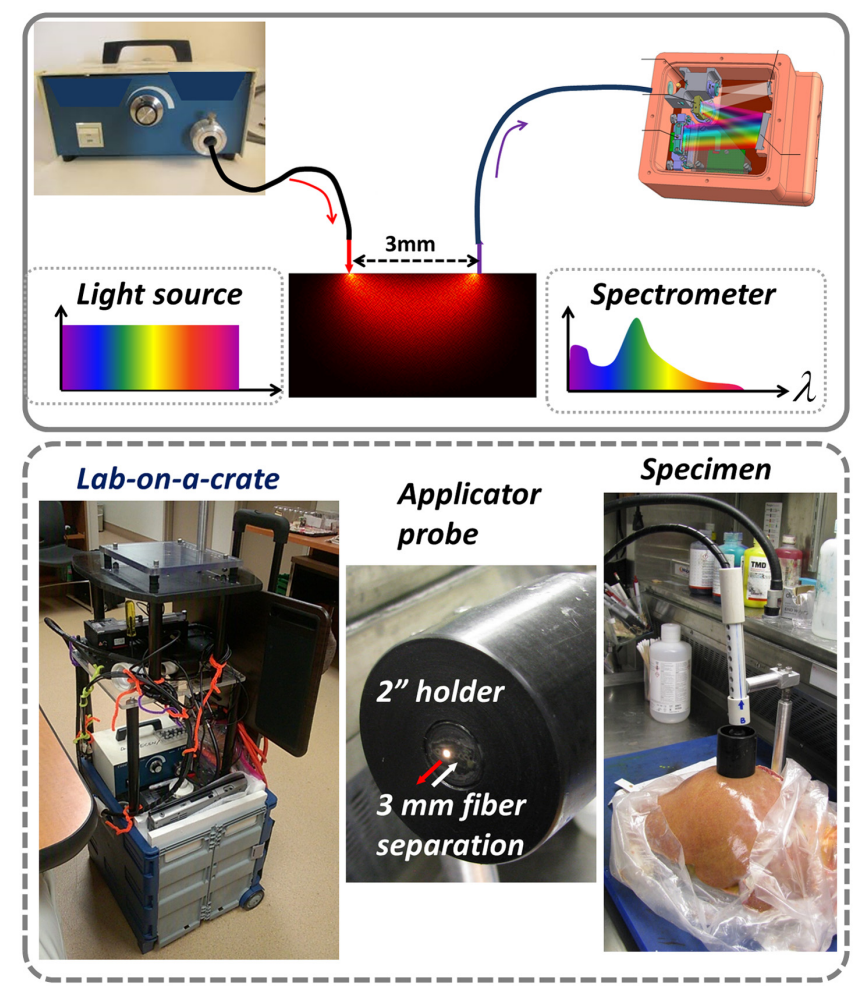

Fig. 4 (Upper panel): Schematic diagram of the DRS system. A light source directs visible/near-infrared light to the tissue via a fiber on the tissue surface. Another fiber at a 3-mm distance from the source fiber collects the diffusely propagated light to a compact spectrometer. (Lower panel) the device built on a wheeled crate (left), the applicator probe (middle), and the hands-free configuration of the applicator probe for measuring liver specimen. 
the ambient light affecting the volume of tissue sampled by the 3-mm separation source-detector pair at the center region of the inner probe having a diameter of $12.5 \mathrm{~mm}$ and (2) to align easily and reliably with a solid phantom of known properties for acquisition of a reference DRS profile. The handheld probe of 2 in. in diameter was also positioned on a platform for the probe to descend by its own weight onto a tissue specimen for hands-free contact with the specimen.

A reference DRS spectral profile, denoted as $I_{\text {phantom }}(\lambda)$, was acquired from a solid phantom (PB0335, INO, Québec, Canada) having an absorption coefficient of $0.021 \mathrm{~mm}^{-1}$ and a reduced scattering coefficient of $1.08 \mathrm{~mm}^{-1}$ at $800 \mathrm{~nm}$. A noise spectral profile, denoted as $I_{\text {noise }}(\lambda)$, was acquired by placing the 2 in. handheld probe on a dark cloth under normal ambient light. The raw DRS spectral profile acquired from a tissue as denoted by $I_{\text {raw }}(\lambda)$ was postprocessed according to the following equation

$R_{\text {tissue }}(\lambda)=\frac{I_{\text {raw }}(\lambda)-I_{\text {noise }}(\lambda)}{I_{\text {phantom }}(\lambda)-I_{\text {noise }}(\lambda)}$,

to deduce a device-specific tissue diffuse reflectance spectral profile $R_{\text {tissue }}(\lambda)$ that is free of the source spectral variation. The effective spectral response of the system was approximately 350 to $1050 \mathrm{~nm}$. All DRS measurements were performed at a 100-ms acquisition time controlled using the vendor-provided graphical user interface of the compact spectrometer.

The DRS study on the liver specimens was approved by the University of Oklahoma Health Sciences Center (OUHSC, IRB \#8155). Within $24 \mathrm{~h}$ of cross clamp, livers not meeting the criteria for transplantation based on information including patient clinical history, warm ischemia time, or gross appearance were measured using the hands-free DRS surface probe in the clinical pathology lab one floor below the Surgery Floor of the University of Oklahoma Medical Center of OUHSC. The DRS measurements were obtained from a random site on the anterior surface of the right lobe and a cut section of the right lobe. The probe was topically cleaned using a swab saturated with $70 \%$ isopropyl alcohol before measuring on a different surface of a specimen. The DRS measurement from each site was the result of averaging over five data acquisitions of 100-ms exposure time each. A total of 10 livers were measured, resulting in 50 combined DRS measurements from the anterior surfaces of the right lobes that were averaged to generate one DRS profile representing DRS measured on the surface of an intact liver. Another set of 50 DRS measurements from the cut-sections of the right lobes of the same 10 livers were averaged to generate one DRS profile specific to measuring on the liver parenchyma in the absence of the liver capsule. Direct DRS measurement on the liver parenchyma immediately adjacent to the capsule was not attempted due to the difficulty of removing only the extremely thin liver capsule.

The kidney specimens were measured by DRS under the same protocol (IRB \#8155). Within $24 \mathrm{~h}$ of cross clamp, kidneys not used for transplantation based on information including patient clinical history, warm ischemia time, gross appearance, and duration of preservation were measured. The DRS measurements were obtained from a random site on the anterior surface of the upper pole of a kidney, and the surface of the upper cortex after the capsule was removed. The DRS measurement from each site was the result of averaging over five data acquisitions of 100-ms exposure time each. A total of nine kidneys were measured, resulting in 45 combined DRS measurements from the anterior surfaces of the upper poles that were averaged to generate a DRS profile corresponding to measuring on the surface of an intact kidney. Another set of 45 DRS measurements from the capsule-removed upper cortex of the same nine kidneys were averaged to generate a DRS profile specific to measuring directly on the kidney parenchyma in the absence of the capsule.

\section{Results}

Examples of the MC simulation outcomes and the model predictions of the DRS over an SDS ranging from 0.01 to $10 \mathrm{~mm}$ at three configurations of the composite media containing the superficial layer and the main medium are shown in Fig. 5. In this figure and all subsequent figures, the measurements produced by MC simulations are plotted using discrete markers, and the model predictions are plotted with solid or dashed lines. The lowest pair of the profiles seeing at the right side of the Fig. 5 corresponds to the DRS when the superficial layer is $1000-\mu \mathrm{m}$ thick and has an absorption coefficient of $\mu_{a 1}=1.0 \mathrm{~mm}^{-1}$, with all other parameters of the superficial layer and the main medium kept at the baseline values, i.e., $n_{[0,1]}=1.40, g_{[0,1]}=0.90, \quad \mu_{a 0}=0.01 \mathrm{~mm}^{-1}$, and $\mu_{s[0,1]}^{\prime}=$ $1.0 \mathrm{~mm}^{-1}$. The $\mu_{a 1}=1.0 \mathrm{~mm}^{-1}$ represents a superficial layer having a contrast of 100 of the absorption coefficient over the main medium and the absorption as strong in magnitude as the reduced scattering $\left(\mu_{a 1}=\mu_{s 1}^{\prime}\right)$. The highest pair of the profiles seeing at the left side of Fig. 5 corresponds to the DRS when the superficial layer is $100-\mu \mathrm{m}$ thick and has a reduced scattering coefficient of $\mu_{s 1}^{\prime}=10 \mathrm{~mm}^{-1}$, with all other parameters of the superficial layer and the main medium kept at the baseline values, i.e., $n_{[0,1]}=1.40, g_{[0,1]}=0.90$, $\mu_{a[0,1]}=0.01 \mathrm{~mm}^{-1}$, and $\mu_{s 0}^{\prime}=1.0 \mathrm{~mm}^{-1}$. The $\mu_{s 1}^{\prime}=10 \mathrm{~mm}^{-1}$ represents a superficial layer having a contrast of 10 of the reduced scattering coefficient over the main medium. The other pair of the profiles in Fig. 5 corresponds to the baseline DRS at the absence of the effect of the superficial layer, i.e., when all parameters of the superficial layer and the main medium are kept at the baseline values, i.e., $n_{[0,1]}=1.40$, $g_{[0,1]}=0.90, \quad \mu_{a[0,1]}=0.01 \mathrm{~mm}^{-1}, \quad$ and $\mu_{s[0,1]}^{\prime}=1.0 \mathrm{~mm}^{-1}$. These values are typical to soft biological tissues. ${ }^{34}$ These baseline DRS profiles are also identical to those MC simulation results, and model prediction profiles presented in Ref. 33 for the same medium properties. The vertical dashed line at the right side of the figure identifies the " $3 \mathrm{~mm}$ " SDS of the handheld probe for measuring the liver and kidney specimens. Some of the MC simulation and model prediction results presented are thus specific to the " $3 \mathrm{~mm}$ " SDS for compatibility with experimental configurations.

\subsection{Effects of the Contrasts of Refractive Index and Anisotropy Factor of a Thin Superficial Layer Over the Main Medium on Spatially Resolved Diffuse Reflectance}

Figure 6 shows the diffuse reflectance when the superficial layer presents various contrast of refractive index or anisotropy factor over the main medium with all other parameters kept at the corresponding baseline values. The results corresponding to varying the refractive index of the superficial layer are presented at the left column, and their counterparts for anisotropy factor at the right column. Any diffuse reflectance profile is normalized with respect to the baseline value corresponding to 


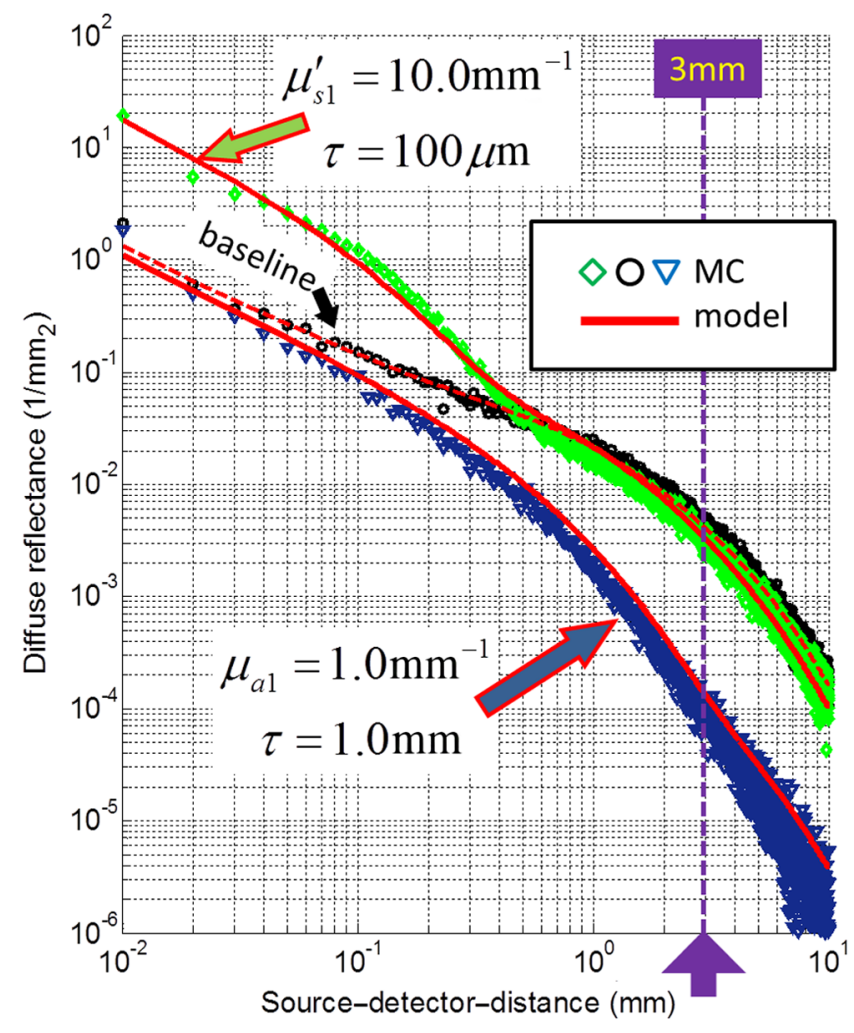

Fig. 5 Examples of the MC simulation outcomes and the model predictions of the DRS over an SDS ranging from $10 \mu \mathrm{m}$ to $10 \mathrm{~mm}$ at three configurations of the composite media containing the superficial layer and the main medium. The lowest pair of the profiles seeing at the right side corresponds to the DRS when the superficial layer is 1$\mathrm{mm}$ thick and has an absorption coefficient of $\mu_{\mathrm{a} 1}=1.0 \mathrm{~mm}^{-1}$, with all other parameters of the superficial layer and the main medium kept at the baseline values. The highest pair of the profiles seeing at the left side corresponds to the DRS when the superficial layer is $100-\mu \mathrm{m}$ thick and has a reduced scattering coefficient of $\mu_{s 1}^{\prime}=10 \mathrm{~mm}^{-1}$, with all other parameters of the superficial layer and the main medium kept at the baseline values. The remaining pair of the profiles corresponds to the baseline DRS at the absence of the effect of the superficial layer. The vertical dashed line at the right side of the figure identifies the " $3 \mathrm{~mm}$ " SDS of the handheld probe for measuring the liver and kidney specimens.

a homogeneous medium of $n_{0}=1.40, g_{0}=0.90, \mu_{a 0}=$ $0.01 \mathrm{~mm}^{-1}$, and $\mu_{s 0}^{\prime}=1.0 \mathrm{~mm}^{-1}$.

The Fig. 6(a) corresponds to the diffuse reflectance at a 3-mm SDS when the superficial layer has a refractive index of 1.30 (circles for MC and the lower solid blue line for model prediction) or 1.50 (diamonds for MC and the upper solid red line for model prediction), versus a main medium refractive index of 1.40, as the superficial layer thickness increases from 10 to $1000 \mu \mathrm{m}$. The pattern of change of the diffuse reflectance estimated by the MC simulations over the range of the superficial layer thickness at a given refractive index of the superficial layer is not clear. The relative magnitude of the diffuse reflectance estimated by the MC simulations between a refractive index of 1.30 and a refractive index of 1.50 of the superficial layer is not clear either. The model-prediction, however, projects a slight reduction $(<5 \%)$ of the diffuse reflectance as the superficial layer thickens at either of the two refractive indices shown, and a slightly higher $(<10 \%)$ diffuse reflectance at superficial layer refractive index of 1.50 than that at 1.30 over the entire range of the superficial layer thickness. The observed changes associated with the refractive index of the superficial layer agree with what can be expected from changing the refractive index as it will cause a slight shift of the positions of the image sources through small modifications to the positions of the respective extrapolated zero-boundary lines. Figure 6(c) corresponds to the diffuse reflectance at a 3-mm SDS when the superficial layer is $10-\mu \mathrm{m}$ thick (circles) or $1000-\mu \mathrm{m}$ thick (diamonds), whereas the refractive index of it increases from 1.30 to 1.50. The pattern of change of the diffuse reflectance estimated by the MC simulations over the range of the superficial layer refractive index is not clear. The model-prediction (solid line for $1000-\mu \mathrm{m}$-thick superficial layer), however, projects a slight elevation $(<10 \%)$ of the diffuse reflectance as the superficial layer refractive index increases from 1.30 to 1.50 at the $1000-\mu \mathrm{m}$ superficial-layer thickness.

Figure 6(b) corresponds to the diffuse reflectance at a 3-mm SDS when the superficial layer has an isotropy factor of 0.80 (circles for MC and the overlapping solid line for model prediction) or 0.99 (diamonds for MC and the overlapping solid line for model prediction), versus the main medium anisotropy factor of 0.90, as the superficial layer thickness increases from 10 to $1000 \mu \mathrm{m}$. The pattern of change of the diffuse reflectance estimated by the MC simulations over the range of the superficial layer thickness is not pronounced. The relative magnitude of the diffuse reflectance estimated by the MC simulations between an isotropy factor of 0.80 and an anisotropy factor of 0.99 of the superficial layer is not pronounced either. The observed patterns that are indifferent between an isotropy factor of 0.80 and an anisotropy factor of 0.99 are expected at the 3-mm SDS as the difference in anisotropy for the same reduced scattering property will be exhausted at a pathlength several times of the reduced scattering pathlength. The model-prediction, however, projects a slight reduction $(<5 \%)$ of the diffuse reflectance as the superficial layer thickens at either of the two isotropic factors shown, and indistinguishable diffuse reflectance values between the superficial layer isotropy factor of 0.99 and 0.80 over the entire range of the superficial layer thickness. Figure 6(d) corresponds to the diffuse reflectance at a 3-mm SDS when the superficial layer is $10-\mu \mathrm{m}$ thick (circles) or $1000-\mu \mathrm{m}$ thick (diamonds), whereas the anisotropy factor of it increases from 0.80 to 0.99 . The pattern of change of the diffuse reflectance estimated by the MC simulations over the range of the superficial layer anisotropy factor is not pronounced. The model-prediction (solid line for 1000- $\mu$ m-thick superficial layer) projects unrecognizable change of the diffuse reflectance as the superficial layer anisotropy factor increases from 0.80 to 0.99 at the $1000-\mu \mathrm{m}$-superficial-layer thickness.

\subsection{Effects of the Contrasts of Absorption Coefficient of a Thin Superficial Layer Over the Main Medium on Spatially Resolved Diffuse Reflectance}

Figure 7 shows the diffuse reflectance when the superficial layer presents various contrast of absorption coefficient over the main medium with all other parameters kept at the corresponding baseline values. The results corresponding to increasing the thickness of the superficial layer when fixing the absorption coefficient of the superficial layer at $1.0 \mathrm{~mm}^{-1}$ are presented at the left column. The results corresponding to increasing the absorption coefficient of the superficial layer at a fixed superficial layer thickness of $1000 \mu \mathrm{m}$ are presented at the right column. All values of the diffuse reflectance are normalized with 

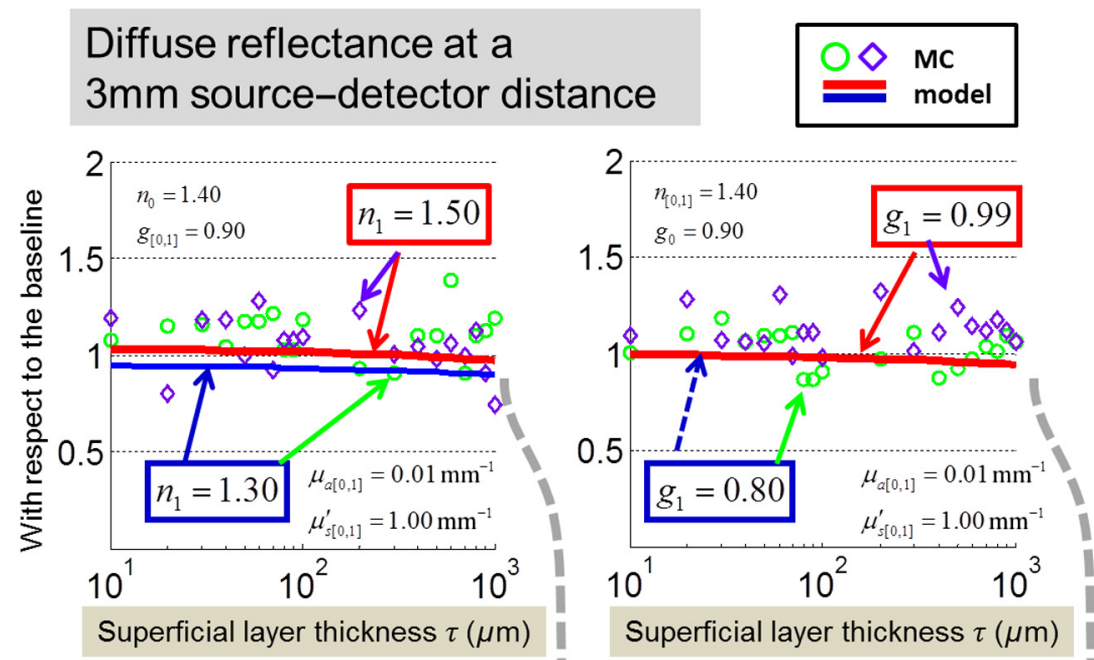

(a)

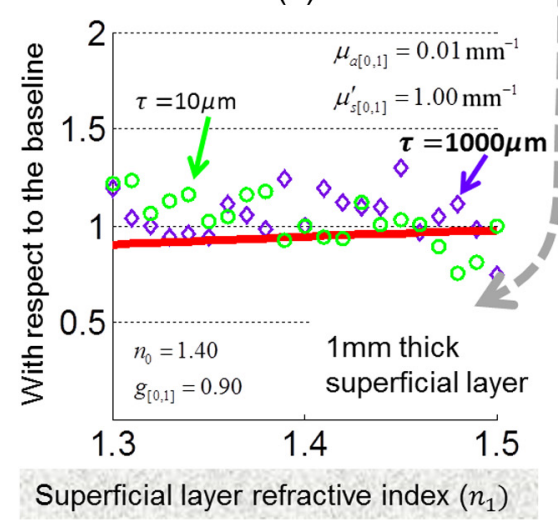

(c)

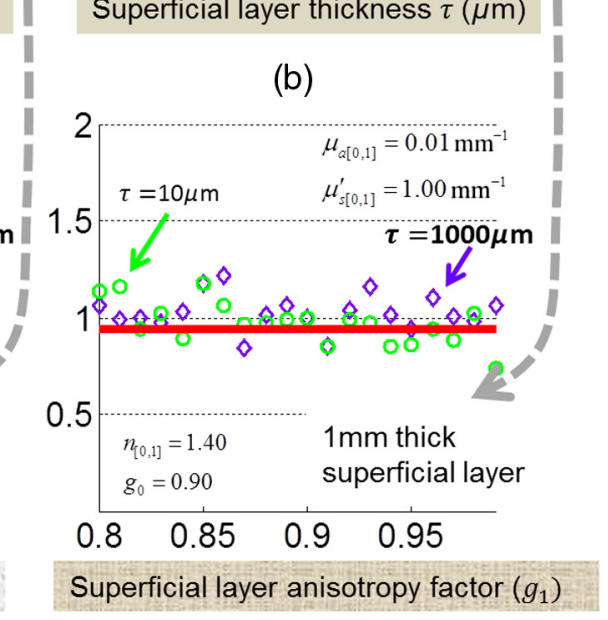

(d)

Fig. 6 The diffuse reflectance when the superficial layer presents various contrast of refractive index or anisotropy factor over the main medium with all other parameters kept at the corresponding baseline values. The results corresponding to varying the refractive index of the superficial layer are presented in (a) and (c), and their counterparts for anisotropy factor in (b) and (d). Any diffuse reflectance profile is normalized with respect to the baseline value. (a) Corresponds to the diffuse reflectance at a 3-mm SDS when the superficial layer has a refractive index of 1.30 or 1.50 , as the superficial layer thickness increases from $10 \mu \mathrm{m}$ to $1 \mathrm{~mm}$. (c) corresponds to the diffuse reflectance at a 3-mm SDS when the superficial layer is $10-\mu \mathrm{m}$ thick or $1-\mathrm{mm}$ thick while the refractive index of it increases from 1.30 to 1.50 . (b) corresponds to the diffuse reflectance at a 3-mm SDS when the superficial layer has an isotropy factor of 0.80 or 0.99 , as the superficial layer thickness increases from $10 \mu \mathrm{m}$ to $1 \mathrm{~mm}$. (d) corresponds to the diffuse reflectance at a 3-mm SDS when the superficial layer is $10-\mu \mathrm{m}$ thick or 1-mm thick while the anisotropy factor of it increases from 0.80 to 0.99 .

respect to their respective baseline values corresponding to a homogeneous medium of $n_{0}=1.40, g_{1}=0.90, \mu_{a 0}=$ $0.01 \mathrm{~mm}^{-1}$, and $\mu_{s 0}^{\prime}=1.0 \mathrm{~mm}^{-1}$.

Figure 7(a) corresponds to the diffuse reflectance at SDSs ranging from 0.01 to $10 \mathrm{~mm}$ when the superficial layer presents a high absorption coefficient of $1.0 \mathrm{~mm}^{-1}$ and the following thicknesses: $10,100,500$, and $1000 \mu \mathrm{m}$. The $\mu_{a 1}=1.0 \mathrm{~mm}^{-1}$ represents a superficial layer having a contrast of 100 of the absorption coefficient over the main medium and the absorption as strong in magnitude as the reduced scattering $\left(\mu_{a 1}=\mu_{s 1}^{\prime}\right)$. The following patterns are identified from (A): (1) for the superficial layer that is more absorbing than the main medium, the deviation of the diffuse reflectance with respect to the baseline value increases as the SDS increases. (2) For the superficial layer that is more absorbing than the main medium, the deviation of the diffuse reflectance with respect to the baseline value increases as the layer thickens. These patterns are all attributed to the increased attenuation of the photons by the superficial layer as it becomes more absorbing or thicker. Figure 7(c) corresponds to the diffuse reflectance at a 3-mm SDS when the superficial layer has an absorption coefficient of $1.0 \mathrm{~mm}^{-1}$, whereas the thickness of it increases from 10 to $1000 \mu \mathrm{m}$. The pattern of the reduction of the diffuse reflectance as projected by the model (solid line) agrees well with that estimated by the MC simulations (diamonds) over the range of the superficial layer thickness. For a superficial layer with an absorption coefficient of $1.0 \mathrm{~mm}^{-1}$ representing 100 times more absorbing than the main medium, a $10-\mu \mathrm{m}$-thick layer will reduce the diffuse reflectance to $\sim 90 \%$, a $100-\mu$ m-thick layer to $\sim 50 \%$, and a $1000-\mu \mathrm{m}$ layer to $<3 \%$ of the baseline value.

Figure 7(b) corresponds to the diffuse reflectance at SDSs ranging from 0.01 to $10 \mathrm{~mm}$ when the superficial layer of 


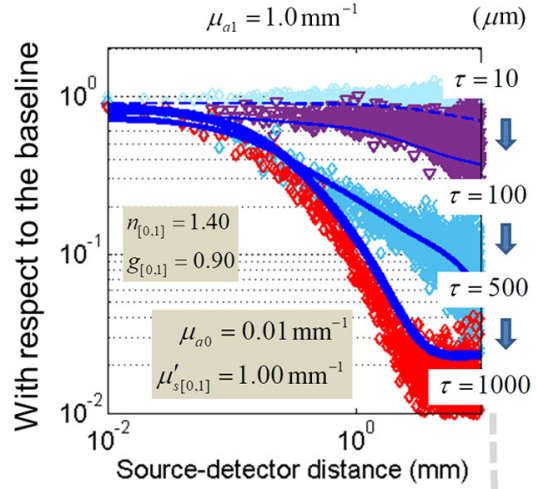

(a)

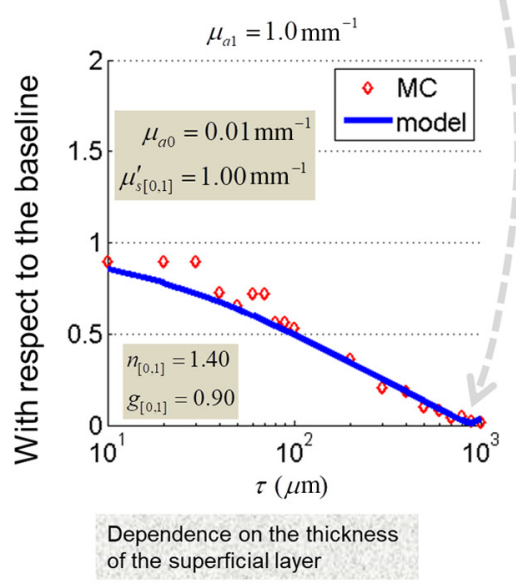

(c)

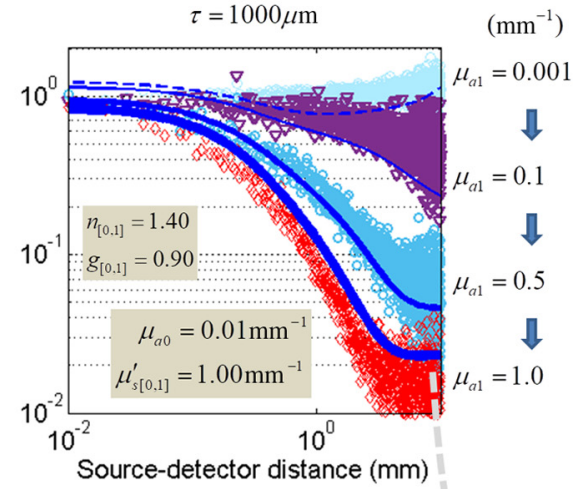

(b)

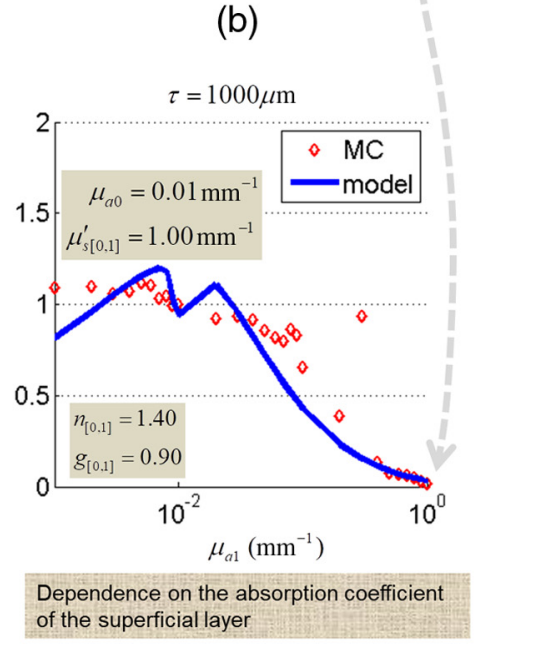

(d)

Fig. 7 The diffuse reflectance when the superficial layer presents various contrast of absorption coefficient over the main medium with all other parameters kept at the corresponding baseline values. All values of the diffuse reflectance are normalized with respect to their respective baseline values. (a) Corresponds to the diffuse reflectance at SDSs ranging from $10 \mu \mathrm{m}$ to $10 \mathrm{~mm}$ when the superficial layer has a high absorption coefficient of $1.0 \mathrm{~mm}^{-1}$ and the following layer thicknesses: 10, 100,500, and $1000 \mu \mathrm{m}$. (c) Corresponds to the diffuse reflectance at a 3-mm SDS when the superficial layer has an absorption coefficient of $1.0 \mathrm{~mm}^{-1}$ while the thickness of it increases from $10 \mu \mathrm{m}$ to $1 \mathrm{~mm}$. (b) Corresponds to the diffuse reflectance at SDSs ranging from $10 \mu \mathrm{m}$ to $10 \mathrm{~mm}$ when the superficial layer of $1-\mathrm{mm}$ thick has the following absorption coefficients: $0.01,0.1,0.5$, and $1.0 \mathrm{~mm}^{-1}$. (d) Corresponds to the diffuse reflectance at a 3-mm SDS when the superficial layer is 1-mm thick while the absorption coefficient of it increases from 0.001 to $1.0 \mathrm{~mm}^{-1}$.

$1000-\mu \mathrm{m}$ thick has the following absorption coefficients: 0.001 , $0.1,0.5,1.0 \mathrm{~mm}^{-1}$, corresponding to $1 / 10,10,50$, and 100 times, respectively, of the absorption coefficient of the main medium. The following patterns are identified from (B): (1) for a $1000-\mu \mathrm{m}$-thick superficial layer, the diffuse reflectance with respect to the baseline value decreases as the absorption coefficient of the superficial layer increases. Figure 7(d) corresponds to the diffuse reflectance at a 3-mm SDS when the superficial layer is $1000-\mu \mathrm{m}$ thick while the absorption coefficient of it increases from 0.001 to $1.0 \mathrm{~mm}^{-1}$. The MC simulations (diamonds) revealed the pattern of the diffuse reflectance that decreases as the superficial layer becomes more absorbing than the main medium. The pattern projected by the model (solid line) agrees with that estimated by MC (diamonds) when the superficial layer is at least three times as absorbing as the main medium. For a $1000-\mu \mathrm{m}$ superficial layer, an absorption coefficient of $0.03 \mathrm{~mm}^{-1}$ of the superficial layer will reduce the diffuse reflectance to $\sim 90 \%$, a $0.2 \mathrm{~mm}^{-1}$ to $<50 \%$, and a $1.0 \mathrm{~mm}^{-1}$ to $<3 \%$ of the baseline value. For the superficial layer that is less than three times as absorbing as the main medium, the pattern projected by the model (solid line) oscillates with respect to the relatively smooth pattern estimated by MC (diamonds), indicating rooms for the model improvement.

\subsection{Effects of the Contrasts of Reduced Scattering Coefficient of a Thin Superficial Layer Over the Main Medium on Spatially Resolved Diffuse Reflectance}

Figure 8 shows the diffuse reflectance when the superficial layer presents various contrast of reduced scattering coefficient over the main medium with all other parameters kept at the corresponding baseline values. The results corresponding to increasing the thickness of the superficial layer when fixing the reduced scattering coefficient of the superficial layer at $10.0 \mathrm{~mm}^{-1}$ are presented at the left column. The results corresponding to 
increasing the reduced scattering coefficient of the superficial layer at a fixed superficial layer thickness of $1000 \mu \mathrm{m}$ are presented at the right column. All values of the diffuse reflectance are normalized with respect to their respective baseline values corresponding to a homogeneous medium of $n_{0}=$ 1.40, $g_{1}=0.90, \mu_{a 0}=0.01 \mathrm{~mm}^{-1}$, and $\mu_{s 0}^{\prime}=1.0 \mathrm{~mm}^{-1}$.

Figure 8(a) corresponds to the diffuse reflectance at SDSs ranging from 0.01 to $10 \mathrm{~mm}$ when the superficial layer with a reduced scattering coefficient of $10.0 \mathrm{~mm}^{-1}$ has the following thicknesses: 10,100 , and $1000 \mu \mathrm{m}$. The $\mu_{s 1}^{\prime}=10 \mathrm{~mm}^{-1}$ represents a superficial layer having a contrast of 10 of the reduced scattering coefficient over the main medium. The following patterns are remarkable: (1) for a superficial layer that is more scattering than the main medium, the diffuse reflectance with respect to the baseline value increases at near-field $(<0.1 \mathrm{~mm})$ but decreases at far-field $(>1 \mathrm{~mm})$. (2) For a superficial layer that is more scattering than the main medium, the deviation of the diffuse reflectance from the baseline value increases as the layer thickens. The second pattern is straightforward to appreciate. The first pattern can be expected qualitatively as the superficial layer becomes more scattering. As the superficial layer picks up scattering, the near-field diffuse reflectance associated with less photon pathlength thus more contribution from the superficial layer will increase, however, the far-field diffuse reflectance associated with deeper photon path will experience a reduction of the photon remission due to more photons escaping the path between the light injection and light detection after the photons are scattered more by the superficial layer. Figure 8(c) corresponds to the diffuse reflectance at a 3-mm SDS when the superficial layer has a reduced scattering coefficient of $10.0 \mathrm{~mm}^{-1}$ while the thickness of it increases from 10 to $1000 \mu \mathrm{m}$. The pattern of the reduction of the diffuse reflectance projected by the model (solid line) agrees globally with that estimated by the MC simulations (diamonds) over the range of the superficial layer thickness. However, the model prediction seems to have underestimated the deviation from the baseline values when the superficial layer is thinner than $60 \mu \mathrm{m}$ and over-estimated the deviation from the baseline values when the superficial layer is thicker than $300 \mu \mathrm{m}$. We note that, the model is not developed as a direct fit to the MC results; therefore, deviations of the model predictions from the MC results are expected when the medium properties or geometries do not strongly support the model basis. For a superficial layer with a reduced scattering coefficient of $10.0 \mathrm{~mm}^{-1}$ representing 10 times more scattering than the main medium, a $10-\mu \mathrm{m}$-thick layer has essentially no effect to the diffuse reflectance at the 3-mm SDS, but a $100-\mu \mathrm{m}$ layer will reduce the diffuse reflectance at the 3-mm SDS to $\sim 90 \%$, and a $1000-\mu \mathrm{m}$ layer to $<25 \%$ of the baseline value, according to the smooth model-prediction.

Figure 8(b) corresponds to the diffuse reflectance at SDSs ranging from 0.01 to $10 \mathrm{~mm}$ when the superficial layer of $1000-\mu \mathrm{m}$ thick has the following reduced scattering coefficients: $0.1,2.0,5.0$, and $10.0 \mathrm{~mm}^{-1}$, corresponding to $1 / 10,2,5$, and 10 times of the reduced scattering coefficient of the main medium. The following patterns are remarkable: (1) For a $1000-\mu \mathrm{m}$-thick superficial layer, the deviation of the diffuse reflectance with respect to the baseline value increases as the reduced scattering coefficient of the superficial layer increases from the baseline value. The subfigure (D) corresponds to the diffuse reflectance at a 3-mm SDS when the superficial layer is $1000-\mu \mathrm{m}$ thick, whereas the reduced scattering coefficient of it increases from 0.1 to $10.0 \mathrm{~mm}^{-1}$. The MC simulations (diamonds) revealed a pattern of the reduction of the diffuse reflectance as the scattering contrast of the superficial layer over the main medium increases but with a lesser extent at a negative contrast of the reduced scattering of the superficial layer over the main medium. The pattern projected by the model (solid line) agrees qualitatively with that estimated by MC (diamonds), but the model prediction seems to have over-estimated the deviation from the baseline values when the superficial layer is less scattering than the main medium and under-estimated the deviation from the baseline values when the superficial layer is more scattering than the main medium. For a $1000-\mu \mathrm{m}$-thick superficial layer, a reduced scattering coefficient of $0.1 \mathrm{~mm}^{-1}$ of the superficial layer will reduce the diffuse reflectance to $\sim 70 \%$, and a $10.0 \mathrm{~mm}^{-1}$ to $<25 \%$ of the baseline value according to the smoother model predictions.

\subsection{Effects of the Absorption and Reduced Scattering Coefficients of the Main Medium on Spatially Resolved Diffuse Reflectance from a Highly Scattering Superficial Layer}

Figure 9 shows the diffuse reflectance at a 3-mm SDS when the superficial layer of $10-$ or $200-\mu \mathrm{m}$ thick is set at a high reduced scattering coefficient of $10.0 \mathrm{~mm}^{-1}$ and the main medium presents various contrast of absorption and reduced scattering coefficient over the superficial layer with other optical parameters kept at the corresponding baseline values. All values of the diffuse reflectance are normalized with respect to the baseline values at a homogeneous medium of $n_{0}=1.40, g_{1}=0.90$, $\mu_{a 0}=0.01 \mathrm{~mm}^{-1}$, and $\mu_{s 0}^{\prime}=1.0 \mathrm{~mm}^{-1}$.

The results in Fig. 9(a) correspond to changing the absorption coefficient of the main medium from 0.001 to $1.0 \mathrm{~mm}^{-1}$ when keeping the superficial layer at an absorption coefficient of $0.01 \mathrm{~mm}^{-1}$ and a reduced scattering coefficient of $10 \mathrm{~mm}^{-1}$. As the absorption of the main medium increases, the diffuse reflectance at the 3-mm SDS reduces monotonically. When the superficial layer having a reduced scattering coefficient of $10.0 \mathrm{~mm}^{-1}$ is $10-\mu \mathrm{m}$ thick, the diffuse reflectance at the 3 -mm SDS is $\sim 115 \%$ of the baseline value for a main medium absorption coefficient of $0.001 \mathrm{~mm}^{-1}$, and reduces monotonically to $<3 \%$ of the baseline value for a main medium absorption coefficient of $1.0 \mathrm{~mm}^{-1}$. When the superficial layer having a reduced scattering coefficient of $10.0 \mathrm{~mm}^{-1}$ is $200-\mu \mathrm{m}$ thick, the diffuse reflectance at the $3-\mathrm{mm}$ SDS is $\sim 75 \%$ of the baseline value for a main medium absorption coefficient of $0.001 \mathrm{~mm}^{-1}$, and reduces monotonically to $<3 \%$ of the baseline value when the main medium absorption coefficient reaches $1.0 \mathrm{~mm}^{-1}$.

The results in Fig. 9(b) correspond to changing the reduced scattering coefficient of the main medium from 0.1 to $10.0 \mathrm{~mm}^{-1}$ when keeping the superficial layer at an absorption coefficient of $0.01 \mathrm{~mm}^{-1}$ and a reduced scattering coefficient of $10 \mathrm{~mm}^{-1}$. As the reduced scattering coefficient of the main medium increases, the diffuse reflectance at the 3-mm SDS reveals a biphasic pattern of increasing initially and decreasing later. This biphasic pattern may be appreciated as the following: as the main medium picks up scattering, more photons reaching the main medium from the superficial layer will be scattered back into the superficial layer after multiple scattering events to cause an initial increase in the diffuse reflectance at the surface of the superficial layer; however, as the main medium continues to pick up scattering, more photons reaching the main medium from the superficial layer will be lost in the main 


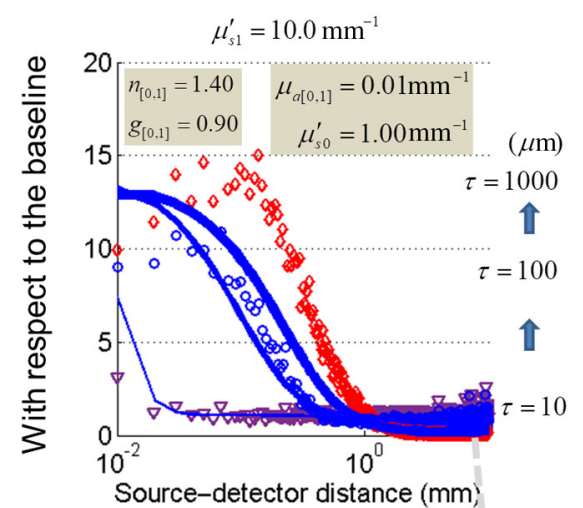

(a)

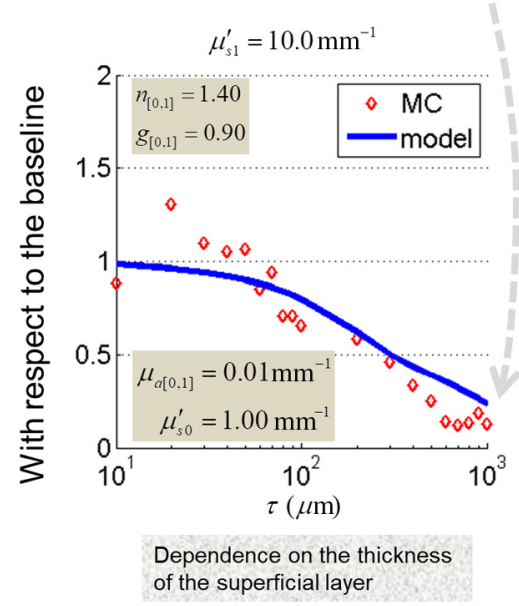

(c)

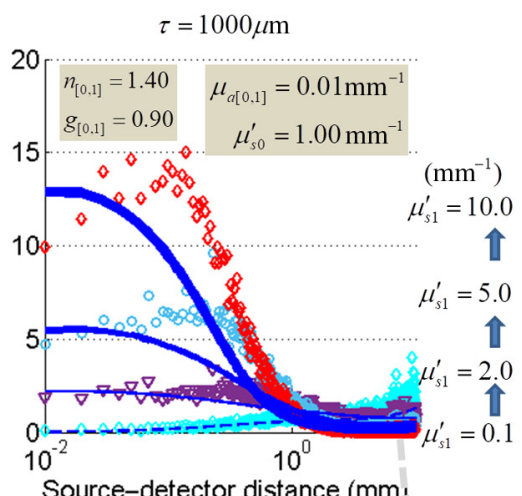

(b)

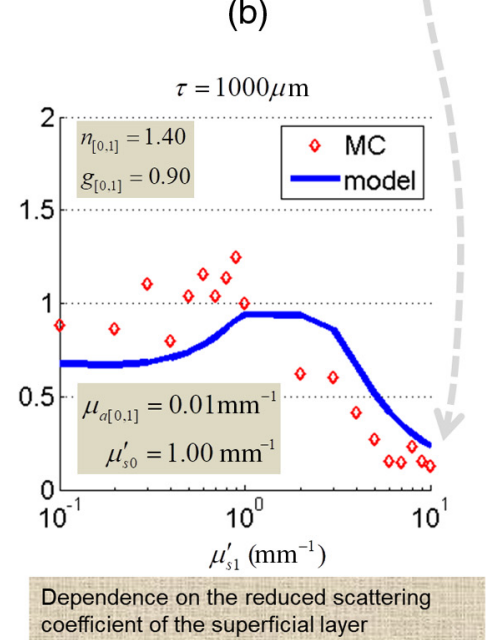

(d)

Fig. 8 The diffuse reflectance when the superficial layer presents various contrast of reduced scattering coefficient over the main medium with all other parameters kept at the corresponding baseline values. All values of the diffuse reflectance are normalized with respect to their respective baseline values. (a) Corresponds to the diffuse reflectance at SDSs ranging from $10 \mu \mathrm{m}$ to $10 \mathrm{~mm}$ when the superficial layer with a reduced scattering coefficient of $10.0 \mathrm{~mm}^{-1}$ has the following layer thicknesses: 10, 100, and $1000 \mu \mathrm{m}$. (c) Corresponds to the diffuse reflectance at a 3-mm SDS when the superficial layer has a reduced scattering coefficient of $10.0 \mathrm{~mm}^{-1}$ while the thickness of it increases from $10 \mu \mathrm{m}$ to $1 \mathrm{~mm}$. (b) corresponds to the diffuse reflectance at SDSs ranging from $10 \mu \mathrm{m}$ to $10 \mathrm{~mm}$ when the superficial layer of 1 -mm thick has the following reduced scattering coefficients: $0.1,2.0,5.0$, and $10.0 \mathrm{~mm}^{-1}$. (d) Corresponds to the diffuse reflectance at a 3-mm SDS when the superficial layer is 1-mm thick while the reduced scattering coefficient of it increases from 0.1 to $10.0 \mathrm{~mm}^{-1}$.

medium due to multiple scattering to cause less photons to be returned to the superficial layer and subsequently a decrease in the diffuse reflectance at the surface of the superficial layer. When the superficial layer having a reduced scattering coefficient of $10.0 \mathrm{~mm}^{-1}$ is $10-\mu \mathrm{m}$ thick, the diffuse reflectance at the $3-\mathrm{mm}$ SDS is $\sim 20 \%$ of the baseline value for a main medium reduced scattering coefficient of $0.1 \mathrm{~mm}^{-1}$, peaks at $\sim 99 \%$ of the baseline value for a main medium reduced scattering coefficient of $1.0 \mathrm{~mm}^{-1}$, then decreases to $\sim 20 \%$ of the baseline value for a main medium reduced scattering coefficient of $10.0 \mathrm{~mm}^{-1}$. When the superficial layer having a reduced scattering coefficient of $10.0 \mathrm{~mm}^{-1}$ is $200-\mu \mathrm{m}$ thick, the diffuse reflectance at the $3-\mathrm{mm}$ SDS is $\sim 5 \%$ of the baseline value for a main medium reduced scattering coefficient of $0.1 \mathrm{~mm}^{-1}$, peaks at $\sim 60 \%$ of the baseline value for a main medium reduced scattering coefficient of $2.0 \mathrm{~mm}^{-1}$, then decreases to $\sim 20 \%$ of the baseline value for a main medium reduced scattering coefficient of $10.0 \mathrm{~mm}^{-1}$.

\subsection{Diffuse Reflectance Spectroscopy of Liver and Kidney with and Without the Capsule}

The device-specific DRS profiles corresponding to measuring on the capsular surface (thicker solid violet line) and subcapsular surface (thicker dashed red line) of a kidney appear below the black line of unity in Fig. 10(a). The device-specific DRS profiles corresponding to measuring on the capsular surface (thinner solid light blue line) and cross section (thinner dashed blue line) of a liver appear globally below the lines corresponding to the kidney in Fig. 10(a). The diffuse reflectance from the liver cross section is lower than that from the subcapsular kidney over 400 to $940 \mathrm{~nm}$, indicating stronger pigment attenuation in the liver than in the kidney if the tissue scattering in the liver can be assumed to be similar to that in the kidney. Figure 10(b) plotted the ratio between the device-specific DRS measured on the capsular surface over that on the subcapsular surface of the kidney (thick solid red line) and the ratio between the 


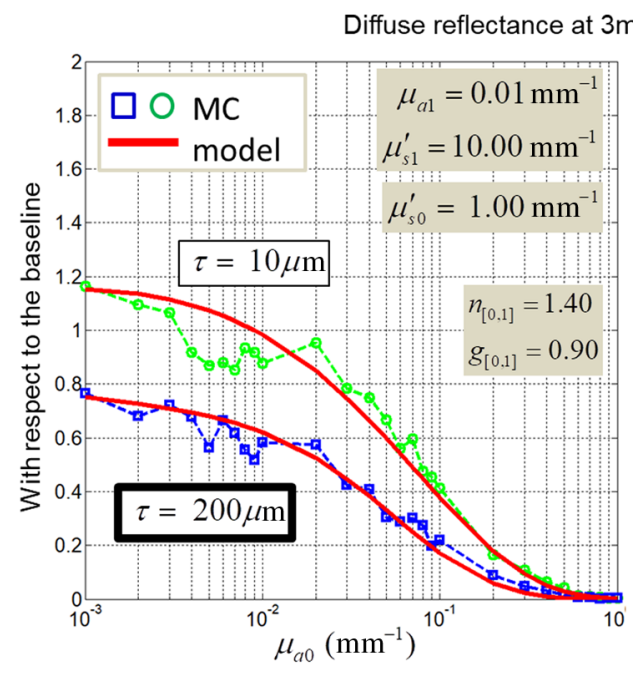

Dependence on the absorption coefficient of the main medium

(a)

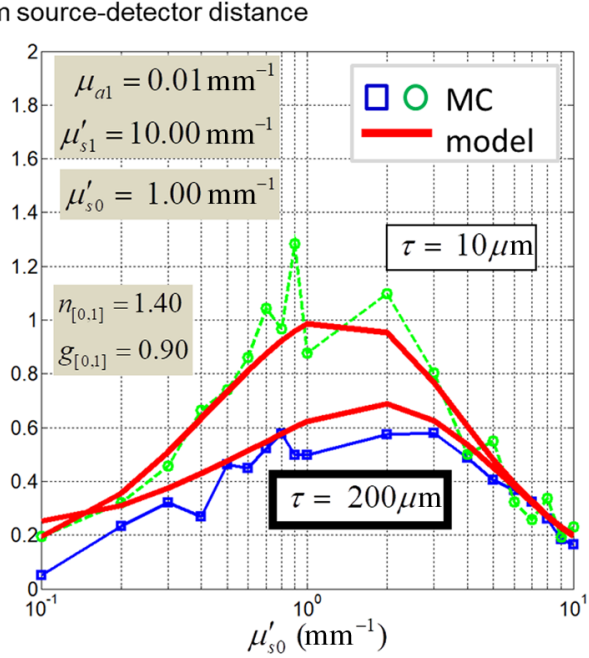

Dependence on the reduced scattering coefficient of the main medium

(b)

Fig. 9 The diffuse reflectance at a 3-mm SDS when the superficial layer of 10- or 200- $\mu \mathrm{m}$ thick is set at a high reduced scattering coefficient of $10.0 \mathrm{~mm}^{-1}$ and the main medium presents various contrast of absorption and reduced scattering coefficient over the superficial layer with other parameters kept at the corresponding baseline values. All values of the diffuse reflectance are normalized with respect to the baseline values. (a) Corresponds to changing the absorption coefficient of the main medium from 0.001 to $1.0 \mathrm{~mm}^{-1}$, when keeping the superficial layer at an absorption coefficient of $0.01 \mathrm{~mm}^{-1}$ and reduced scattering coefficient of $10 \mathrm{~mm}^{-1}$. (b) Corresponds to changing the reduced scattering coefficient of the main medium from 0.1 to $10.0 \mathrm{~mm}^{-1}$ when keeping the superficial layer at an absorption coefficient of $0.01 \mathrm{~mm}^{-1}$ and reduced scattering coefficient of $10 \mathrm{~mm}^{-1}$.

device-specific DRS measured on the capsular surface over that on the cross-section of the liver (thin solid blue line), both appearing above the black line of unity. The ratio corresponding to the kidney varies between 1.01 (or 101\%) around $800 \mathrm{~nm}$ and 1.7 (or $170 \%$ ) that occurs at $\sim 500 \mathrm{~nm}$. The ratio corresponding to the liver varies between 1.05 (or 105\%) around $800 \mathrm{~nm}$ and 1.2 (or $120 \%$ ) that occurs at shorter $(<400 \mathrm{~nm})$ or longer $(>1000 \mathrm{~nm})$ wavelength ranges.

To evaluate that if the much greater difference of the capsular versus subcapsular device-specific DRS in the kidney than in the liver is potentially caused by the much thicker capsule of the kidney than that of the liver, the device-specific DRS for the kidney or liver as plotted in Fig. 10(b) is used to estimate the main medium absorption property, which is then implemented in the analytical model presented earlier to estimate the difference between capsular and subcapsular DRS from a liver or a kidney for a $10-\mu \mathrm{m}$-thick liver capsule and a $200-\mu$ m-thick kidney capsule, as schematically shown in Fig. 10(c). The estimation is conducted according to the following procedures: (1) the spectrum of tissue absorption coefficient was calculated as $\mu_{a}(\lambda)=\left\{\ln \left[R_{\text {tiss }}(\lambda)\right]^{-1}\right\} / 9+\mu_{a_{\text {_phantom }} \text {, }}$ where the number " 9 " is the product of the SDS in mm " 3 " and a differential pathlength factor of " 3 " estimated for the 3 -mm SDS. ${ }^{37}$ The $\mu_{a_{-} \text {phantom }}=0.021 \mathrm{~mm}^{-1}$ is the absorption coefficient of the reference solid phantom. The extracted absorption coefficient spectrum was then assigned as the absorption coefficient spectrum of the main medium. (2) The absorption coefficient spectrum of the capsular layer is estimated as $\mu_{a 1}(\lambda)=0.3 * \lambda^{-0.1}$, where $\lambda$ is the wavelength in $\mu \mathrm{m}$, based on the absorption properties suggested by experimental reports. $^{38}$ (3) The reduced scattering coefficient of the main medium is set as $1.0 \mathrm{~mm}^{-1}$. (4) The reduced scattering coefficient spectrum of the superficial layer is estimated as $\mu_{s 1}^{\prime}(\lambda)=3.2 * \lambda^{-1}$, where $\lambda$ is the wavelength in $\mu \mathrm{m}$, based on the reduced scattering properties suggested by experimental reports. ${ }^{38}(5)$ The afore-estimated spectra were implemented in the analytical model. The ratio of device-specific DRSs between measuring on the kidney parenchyma in the presence of a realistic $200-\mu \mathrm{m}$-thick capsule and in the absence of the capsule is estimated as the ratio between the model-outputs of the diffuse reflectance at a superficial layer thickness of 200 and $0.1 \mu \mathrm{m}$ for a main medium absorption spectrum derived using the kidney measurements. Similarly, the ratio of device-specific DRSs between measuring on the liver parenchyma in the presence of a realistic $10-\mu \mathrm{m}$-thick capsule and in the absence of the capsule is estimated as the ratio between the model outputs of the diffuse reflectance at a superficial layer thickness of 10 and $0.1 \mu \mathrm{m}$ for a main medium absorption spectrum derived using the liver measurements.

The model-prediction of the ratio of the diffuse reflectance from the kidney in the presence of the realistic 200- $\mu$ m capsule over that in the absence of the capsule is shown in Fig. 10(d) as the thick solid red line, after aligning the peak magnitude around the $500 \mathrm{~nm}$ to that of the experimentally measured profile shown in Fig. 10(b). The DRS profile for the kidney shown in (D) based on a realistic $200-\mu \mathrm{m}$ capsule resembles closely that shown in (B), indicating that the ratio between the surface DRS and subcapsular DRS varies significantly over the spectrum of measurements. The ratio corresponding to the kidney as shown in (D) estimated for a realistic $200-\mu \mathrm{m}$ capsule varies between 0.81 (or $81 \%$ ) around $300 \mathrm{~nm}$ and peaks at 1.7 (or $170 \%$ ) that occurs at $\sim 500 \mathrm{~nm}$. The profile corresponding to the kidney estimated with a realistic capsule thickness of $200 \mu \mathrm{m}$ reproduces the experimentally measured profile at $>420 \mathrm{~nm}$ but deviates 


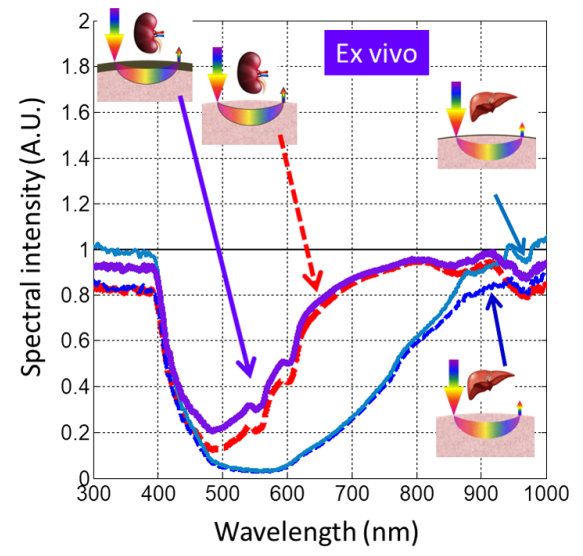

(a)

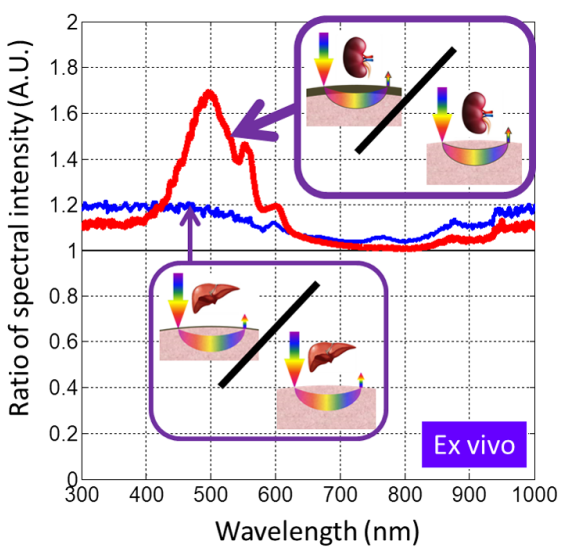

(b)

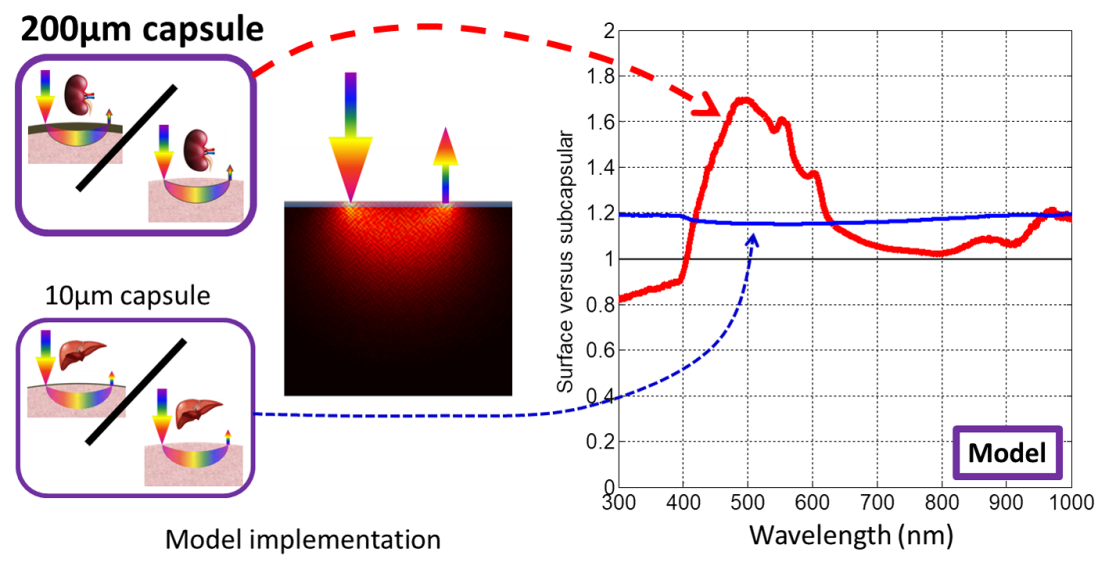

(c)

(d)

Fig. 10 (a) The DRS profile corresponding to measuring on the capsular surface of a kidney is plotted as the thicker solid violet line. The DRS profile corresponding to measuring on the subcapsular surface of a kidney is plotted as the thicker dashed red line. The DRS profile corresponding to measuring on the capsular surface of a liver is plotted as the thinner solid light blue line. The DRS profile corresponding to measuring on the cross section of a liver is plotted as the thinner dashed blue line. (b) The ratio between the DRS measured on the capsular surface over that on the subcapsular surface of a kidney is plotted as thicker solid red line. The ratio between the DRS measured on the capsular surface over that on the cross-section of a liver is plotted as the thinner solid blue line. (c) The model implementation to calculate the ratio between DRS measured on the surface of a kidney and on the subcapsular surface of a kidney, assuming a kidney capsule thickness of $200 \mu \mathrm{m}$. The model implementation to calculate the ratio between DRS measured on the surface of a liver and on the subcapsular parenchyma of a liver, assuming a liver capsule thickness of $10 \mu \mathrm{m}$. (d) The model-predicted ratio of the diffuse reflectance from the kidney in the presence of the realistic $200-\mu \mathrm{m}$ capsule over that in the absence of the capsule is displayed as the thick solid red line. The model-predicted ratio of the diffuse reflectance from the liver in the presence of the realistic $10-\mu \mathrm{m}$ capsule over that in the absence of the capsule is displayed as the thinner solid blue line.

significantly from the experimentally measured profile at $<420 \mathrm{~nm}$. The model-prediction of the ratio of the diffuse reflectance from the liver in the presence of the realistic $10-\mu \mathrm{m}$ capsule over that in the absence of the capsule is shown in Fig. 10(d) as the thinner solid blue line, after aligning the plateau at $<400 \mathrm{~nm}$ with that of the experimentally measured profile shown in Fig. 10(b). The DRS profile for the liver shown in (D) based on a realistic $10-\mu \mathrm{m}$ capsule resembles globally that shown in (B), indicating that the ratio between the surface DRS and subcapsular DRS varies insignificantly over the spectrum of measurements. The ratio corresponding to the liver as shown in (D) estimated for a realistic $10-\mu \mathrm{m}$ capsule varies between 1.17 (or $117 \%$ ) over 500 to $600 \mathrm{~nm}$ and 1.2 (or 120\%) that occurs at shorter $(<400 \mathrm{~nm})$ or longer $(>1000 \mathrm{~nm})$ wavelength ranges.

\section{Discussion}

Diffuse reflectance measurement, which renders the conveniences for noninvasive surface assessment of the optical properties of a parenchyma, should be performed judiciously on an organ in the presence of capsule. As is shown in Fig. 10(a), the device-specific DRS profile of the kidney parenchyma varies over a smaller scale in the 400- to 950-nm range when compared with that of the liver parenchyma. However, in regard to the difference between DRS on the organ in the presence of capsule and DRS on capsule-free parenchyma, the spectral variance of the DRS corresponding to the kidney is much greater (101 to $170 \%$ ) than that of the liver (105 to $120 \%)$, as shown in Fig. 10(b). The relatively small spectral variation of the DRS on the capsular surface of the liver over the DRS on the 
cross-section of the liver when measured at the 3-mm SDS indicates that using the surface DRS measured at the 3-mm SDS on the liver to estimate the optical properties of the subcapsular parenchyma may be a safe approach. However, if the SDS reduces significantly, e.g., to a few hundreds of microns, the effect of even the thin capsular layer of the liver on the surface DRS could increase according to the patterns revealed by the MC simulations. For the kidney however, even at the 3-mm SDS, the spectral difference between the DRS on the capsular surface and the DRS on the subcapsular parenchyma is relatively large, suggesting that the optical properties of the subcapsular kidney parenchyma estimated using the surface DRS measured at the 3-mm SDS on the kidney will deviate spectrally from the true conditions of the subcapsular parenchyma.

The model-predicted spectral variations between DRS measured on the liver in the presence of the capsule and in the absence of the capsule as shown in Fig. 10(d) agree with the experimentally measured patterns at the shorter $(<400 \mathrm{~nm})$ and longer $(>950 \mathrm{~nm})$ wavelength regimes as shown in Fig. 10(b). The model-implementation predicted a much smoother profile from 400 to $950 \mathrm{~nm}$ for the liver when compared with the experimentally measured profile in the same spectral range. It is worth noting that, the model implementation using the DRS that was experimentally measured from the subcapsular or cross-sectional parenchyma has some limiting constraints applying to both the liver and kidney, including the use of differential path length, the absence of spectral dependence of the reduced scattering of the parenchyma, simplification of the absorption properties of the capsular layer as following a weak inverse dependence on the wavelength, and neglecting the spectral dependences of the absorption and reduced scattering of the reference phantom. The simplifications of the model-implementation caused the model-prediction to miss the spectral variation details of the liver profile shown by the experimental measurements. The simplifications of the model-implementation has also caused the model-prediction for kidney to deviate significantly from the experimental measurements at the $<420-\mathrm{nm}$ range. The relatively flat profile of the ratio between surface DRS and subcapsular DRS from the kidney at $<420 \mathrm{~nm}$ suggests that the spectral properties of the kidney capsule at the $<420$-nm range are quite similar to those of the kidney parenchyma. The spectral profile corresponding to experimental measurements from the kidney also has shown a near-identity value of $101 \%$ near $800 \mathrm{~nm}$; surface measurement at this wavelength is expected to closely represent the subcapsular parenchyma. However, as diffuse reflectance from the kidney (or other organ) will rely on spectral information to reveal parenchyma functional or pathological conditions, it is necessary to correct the capsular effect to the surface measurements when the spectrum of operation is broader than the limited capsule-insensitive wavelengths.

Despite that the model-implementation has missed some details of the spectral variations between the surface DRS and parenchyma DRS for the liver, there is a good global agreement of the model predictions with the experimental measurements regarding how the surface DRS of the parenchyma is affected by the thickness of a highly scattering capsule. According to Fig. 9 wherein the model and data agree upon a main medium underlying a highly scattering superficial layer, we expect the following: (1) if the main medium develops more pigments such as increased vasculature causing an increase in the absorption coefficient, the surface DRS will decrease; (2) if the main medium becomes more scattering, the surface DRS will increase initially than decrease later as the scattering keeps increasing. Such biphasic change of the surface DRS in response to the change of parenchymal scattering properties may be particularly important to interpreting the surface DRS from livers with fibrotic injury. As the fibrosis develops in the liver, the scattering of the hepatic parenchyma is expected to increase, which may cause the surface DRS signal to increase at a lower grade of fibrosis but decrease with increased level of fibrosis pathology. If the hepatic parenchyma presents pathology conditions other than fibrosis, such as necrosis or steatosis, the profile change of the surface DRS from the liver as the fibrosis develops will become more complicated.

A limitation to the $\sim 1760 \mathrm{MC}$ simulations in this work was the execution of each MC simulation at a total photon number of 100,000 that is substantially smaller than the number implemented by many other MC studies when evaluating the diffuse reflectance from a medium of two-layer geometry. ${ }^{26-32,39,40}$ As a result, the MC outputs in this work carried much greater variations than those appearing in other MC studies. Those variations carried by the MC outputs that were caused by relatively low amount of photons would, however, be equivalent to noises common to experimental measurements. The relatively noisy MC simulation results, thus, have made a realistic data context for the model prediction to compare against. Regardless of how noisy the MC simulations could have been, the patterns of changes, specifically the stronger deviation from the baseline set when the superficial layer is thicker or has a greater contrast of the optical properties over the main medium, are expected to be retained in the $\mathrm{MC}$ results.

The MC results have revealed that surface DRS of a main medium covered by a capsular layer is quite insensitive to the contrast of the refractive index or the anisotropy factor between the capsular layer and the main medium. The indices of refraction of most soft tissues lie within the 1.38 to 1.41 range, with the exception of adipose tissue, which has a refractive index of $\sim 1.47 .{ }^{41}$ A recent study evaluating diffuse reflectance from a medium with multiple layers ${ }^{42}$ reported that a mismatch in the interlayer refractive indices or anisotropy factors has only a slight effect on the magnitude of the intensities of the diffuse reflectance. The effect of a capsular layer to DRS is expected to be even smaller under an index-matched condition. ${ }^{43}$ These earlier observations of the insensitivity of the diffuse reflectance on the index-of-refraction variations are confirmed by the results of this work.

The geometry presented in this work is limited to an otherwise homogeneous medium "perturbed" by a thin superficial layer. The deviations of the contributions to the surface DRS are modeled as being caused by an addition to the slave source that becomes stronger as the capsular layer gains either the optical contrast over the main medium or the thickness. The thin superficial layer that becomes heterogeneous with respect to the main medium not only changes the slave source contribution to the photon irradiation but also alters the master source contribution. The combined deviation of the master and slave contribution to the photon irradiation from their respective values of the corresponding homogeneous medium is lumped into a differential contribution to the photon irradiation that vanishes as the capsule layer becomes negligible in optical properties or 
thickness. The analytical model justifies the effect of the medium property change on DRS with a set of contributions that deviate from their respective values corresponding to a homogeneous medium. The analytical model shown to resolve the salient features of the effect of a thin superficial layer ( $<1$-mm thickness) on surface DRS of the underlying main medium could be extended to the geometry of a two-layered medium with a superficial layer that is much thicker than $1 \mathrm{~mm}$. It can be expected that, applying the master-slavedifferential source approach to a two-layer medium containing a thick surface layer may require the differential source to modulate more of the master source than the slave as is applying to the effect of thin superficial layer of this present work. The analytical model shown to apply to a thin superficial layer $(<1-\mathrm{mm}$ thickness) overlaying the main medium may also be useful to the geometry of more than two layers, for which perhaps multiple differential sources with different moments may become necessary. We also note that, the analytical framework presented in this work shall not be limited to understanding the effect of capsule to steady-state diffuse reflectance. Extension of the analytical approach is planned for addressing frequency-domain diffuse reflectance in the presence of a thin surface layer ${ }^{44}$ and time-resolved diffuse reflectance in the presence of a capsular layer. ${ }^{32,45}$ The rendering of an analytical model may also allow exploring if DRS using some convenient configurations such as the isopathlength point discovered for a homogeneous medium ${ }^{46}$ is possible in the presence of a thin superficial layer atop the homogeneous medium.

\section{Conclusion}

The capsular optical properties and thickness combined are projected to affect how accurate the diffuse reflectance on the surface of a capsular solid organ represents that on the subcapsular parenchyma. The projection of the combined effect of the contrast of the optical properties over the main medium and the thickness of the capsular layer is supported by MC simulations and modeled by an analytical method. The MC simulations are implemented for two-layer geometries containing a thin superficial layer with the thickness from $10 \mu \mathrm{m}$ to $1 \mathrm{~mm}$ to evaluate the surface diffuse reflectance over a source-detector separation spanning $10 \mu \mathrm{m}$ to $10 \mathrm{~mm}$. The superficial layer was set to have various contrasts concerning refractive index, anisotropy factor, absorption coefficient, and reduced scattering coefficient, versus those of the subsurface main medium. An analytical approach by modifying a master-slave dual-source setting to a masterslave-differential triple-source configuration was proposed to account for how the superficial layer of various thicknesses and optical properties affect surface diffuse reflectance. DRS was also performed ex vivo on 10 fresh human livers and 9 fresh human kidneys. The device-specific diffuse reflectance performed on the capsular surface was as high as $170 \%$ of that on the subcapsular parenchyma in the kidney. The device-specific diffuse reflectance performed on the capsular surface was as high as $120 \%$ of that on the cross-sectional parenchyma in the liver. According to the analytical model, the significantly greater spectral deviation of surface diffuse reflectance between with and without the capsule in the kidney than in the liver was accountable by the much thicker capsule of the kidney than the liver.

\section{Appendix A}

The following notations apply to all components of photon irradiation:

$\operatorname{sign}\left(\mu_{s 1}^{\prime}\right)= \begin{cases}1 & \text { if } \mu_{s 1}^{\prime} \geq \mu_{s 0}^{\prime} \\ -1 & \text { if } \mu_{s 1}^{\prime}<\mu_{s 0}^{\prime}\end{cases}$ $[0,1]$

$\delta=\frac{\tau}{z_{\text {caps }}}$ is the dimensionless thickness of the capsule, range $\Delta_{a}=\left(\frac{\mu_{a 1}-\mu_{a 0}}{\mu_{a 1}+\mu_{a 0}}\right)^{2}$ is the index of contrast for absorption.

$\Delta_{s^{\prime}}=\left(\frac{\mu_{s 1}^{\prime}-\mu_{s 0}^{\prime}}{\mu_{s 1}^{\prime}+\mu_{s 0}^{\prime}}\right)^{2}$ is the index of contrast for reduced scattering.

\section{A.1 Parameters Applying to Only the Master Component of the Photon Irradiation}

$$
\begin{aligned}
& n^{\text {master }}=n_{0} \text { (refractive index of the main medium) } \\
& g^{\text {master }}=g_{0} \text { (anisotropy factor of the main medium) } \\
& \mu_{a}^{\text {master }}=\mu_{a 0} \text { (absorption coefficient of the main medium) } \\
& \mu_{s^{\prime}}^{\text {master }}=\mu_{s 0}^{\prime} \text { (reduced scattering coefficient of the main } \\
& \text { medium) } \\
& D^{\text {master }}=D_{0} \text { (diffusion coefficient of the main medium) } \\
& \mu_{s}^{\text {master }}=\mu_{s 0} \text { (scattering coefficient of the main medium) } \\
& \xi^{\text {master }}=\xi_{0} \text { (refractive index mismatch index) } \\
& A_{0 / 1}=\frac{1+\xi_{0 / 1}}{1-\xi_{0 / 1}} \text { (zero-boundary extrapolation factor) } \\
& \eta^{\text {master }}=\eta_{0} \text { (slave-source index determined by the properties } \\
& \text { of the main medium) } \\
& z_{a}^{\text {master }}=z_{a 0} \text { (master source depth determined by the }
\end{aligned}
$$

\section{A.2 Parameters Applying to Only the Slave Component of the Photon Irradiation}

$$
\begin{aligned}
n^{\text {slave }}= & \left.n_{1} \text { (refractive index of the superficial layer }\right) \\
g^{\text {slave }}= & g_{1}(\text { anisotropy factor of the superficial layer } \\
\varepsilon_{a \& s^{\prime}}^{\text {slave }}= & 1-\left[1-\left(1-\Delta_{a}\right)^{\alpha} \cdot\left(1-\Delta_{s}^{\prime}\right)^{\beta}\right] \cdot \delta^{\gamma}, \text { where }(\alpha, \beta, \gamma)= \\
& (2,2,1) \\
\mu_{a}^{\text {slave }}= & \mu_{a 1}-\left(\mu_{a 1}-\mu_{a 0}\right) \varepsilon_{a \& s^{\prime}}^{\text {slave }}(\text { slave absorption coefficient }) \\
\mu_{s^{\prime}}^{\text {slave }}= & \mu_{s 1}^{\prime}-\left(\mu_{s 1}^{\prime}-\mu_{s 0}^{\prime}\right) \varepsilon_{a \& s^{\prime}}^{\text {slave }} \quad \text { (slave reduced scattering } \\
& \text { coefficient }) \\
D^{\text {slave }}= & \frac{1}{3\left(\mu_{a}^{\text {slave }}+\mu_{s^{\prime}}^{\text {slave }}\right)}(\text { slave diffusion coefficient }) \\
\mu_{s}^{\text {slave }}= & \left.\frac{\mu_{s}^{\text {slave }}}{g^{\text {slave }}} \text { (slave scattering coefficient }\right)
\end{aligned}
$$


$\xi^{\text {slave }}=-1.440\left(n^{\text {slave }}\right)^{-2}+0.710\left(n^{\text {slave }}\right)^{-1}+0.668+$ $0.0636\left(n^{\text {slave }}\right)$ (slave refractive index mismatch index)

$A^{\text {slave }}=\frac{1+\xi^{\text {slave }}}{1-\xi_{\text {slave }}}($ slave zero-boundary extrapolation index $)$

$\eta^{\text {slave }}=\left[g^{\text {slave }} \cdot \exp \left(1-g^{\text {slave }}\right)\right]^{1 / 10}$ (slave-source index $)$

$z_{a}^{\text {slave }}=\left[\frac{1}{\mu_{s}^{\text {slave }}}\right]^{2} \cdot \mu_{s^{\prime}}^{\text {slave }} \cdot\left(1-\eta^{\text {slave }}\right)$ (slave-source depth)

$z_{b}^{\text {slave }}=2 \cdot A^{\text {slave }} \cdot D^{\text {slave }}$ (slave zero-boundary offset)

$l_{\text {real }}^{\text {slave }}=\sqrt{\rho^{2}+\left(z_{a}^{\text {slave }}\right)^{2}}$ (distance from the slave-source to the detector)

$l_{\text {imag }}^{\text {slave }}=\sqrt{\rho^{2}+\left(z_{a}^{\text {slave }}+2 \cdot z_{b}^{\text {slave }}\right)^{2}}$ (distance from the image of the slave source to the detector)

$\theta_{a}^{\text {slave }}=\exp \left[-\frac{1}{4}\left(\frac{\mu_{a 1}}{\mu_{a 0}}\right)^{2}\right]$ (absorption correction factor)

$\theta_{s^{\prime}}^{\text {slave }}=1-\exp \left[-2\left(\frac{\mu_{s 1}^{\prime}}{\mu_{s 0}^{\prime}}\right)^{2}\right]$ (scattering correction factor)

$\varepsilon_{\mathrm{eff}}^{\text {slave }}=1-\left[1-\left(1-\Delta_{a}\right)^{\alpha} \cdot\left(1-\Delta_{s}^{\prime}\right)^{\beta}\right] \cdot \delta^{\gamma}$, where $(\alpha, \beta, \gamma)=$ $(1 / 100,2,1)$

$\mu_{\text {eff }}^{\text {slave }}=\sqrt{\frac{\mu_{a}^{\text {slave }}}{D^{\text {slave }}}} \frac{1}{\varepsilon_{\text {eff }}^{\text {slave }}}($ slave effective attenuation coefficient $)$

$\varepsilon_{\text {strength }}^{\text {slave }}=1-\left[1-\left(1-\Delta_{a}\right)^{x} \cdot\left(1-\Delta_{s}^{\prime}\right)^{y}\right] \cdot \delta^{z}, \quad$ where $(x, y, z)=(1 / 100,2,1 / 8)$

$S^{\text {slave }}=S^{\text {master }} \cdot\left[S_{1}^{*}-\left(S_{1}^{*}-S_{0}^{*}\right) \cdot \varepsilon_{\text {strength }}^{\text {slave }}\right]$. $\frac{\theta_{s^{\prime}}^{\text {slave }}}{\left[\left(\varepsilon_{\text {strength }}^{\text {slave }}\right)^{1 / 10}\right]^{\operatorname{sign}\left(\mu_{s}^{\prime}\right)}\left[1-\Delta_{a} \cdot \delta\right]^{1 / 2}}$ (slave-source strength)

\section{A.3 Parameters Applying to the Differential Component of the Photon Irradiation}

$\varepsilon_{n \& g}^{\text {diff }}=1-\left[1-\left(1-\Delta_{a}\right)^{\alpha} \cdot\left(1-\Delta_{s}^{\prime}\right)^{\beta}\right] \cdot \delta^{\gamma}$, where $(\alpha, \beta, \gamma)=$ $(2,2,0)$

$n^{\text {diff }}=n_{1}-\left(n_{1}-n_{0}\right) \varepsilon_{n \& g}^{\text {diff }}($ differential refractive index $)$

$g^{\text {diff }}=g_{1}-\left(g_{1}-g_{0}\right) \varepsilon_{n \& g}^{\text {diff }}$ (differential anisotropy factor)

$\varepsilon_{a \& s^{\prime}}^{\text {slave }}=1-\left[1-\left(1-\Delta_{a}\right)^{\alpha} \cdot\left(1-\Delta_{s}^{\prime}\right)^{\beta}\right] \cdot \delta^{\gamma}$, where $(\alpha, \beta, \gamma)=$ $(2,2,1)$

$\mu_{a}^{\text {diff }}=\mu_{a 1}-\left(\mu_{a 1}-\mu_{a 0}\right) \varepsilon_{a \& s^{\prime}}^{\text {diff }} \quad$ (differential absorption coefficient)

$\mu_{s^{\prime}}^{\text {diff }}=\mu_{s 1}^{\prime}-\left(\mu_{s 1}^{\prime}-\mu_{s 0}^{\prime}\right) \varepsilon_{a \& s^{\prime}}^{\text {diff }}($ differential reduced scattering coefficient)

$D^{\text {diff }}=\frac{1}{3\left(\mu_{a}^{\text {diff }}+\mu_{s^{\prime}}^{\text {diff }}\right)}($ differential diffusion coefficient $)$

$\mu_{s}^{\text {diff }}=\frac{\mu_{s^{\prime}}^{\text {diff }}}{g^{\text {diff }}}($ differential scattering coefficient $)$

$\xi^{\mathrm{diff}}=-1.440\left(n^{\mathrm{diff}}\right)^{-2}+0.710\left(n^{\mathrm{diff}}\right)^{-1}+0.668+0.0636\left(n^{\mathrm{diff}}\right)$ (differential refractive index mismatch index)

$A^{\text {diff }}=\frac{1+\xi_{\text {diff }}}{1-\xi_{\text {diff }}}($ differential zero-boundary extrapolation factor) $\eta^{\text {diff }}=\left[g^{\text {diff }} \cdot \exp \left(1-g^{\text {diff }}\right)\right]^{1 / 10}($ differential source index $)$ $z_{a}^{\text {diff }}=\left[\frac{1}{\mu_{s}^{\text {diff }}}\right]^{2} \cdot \mu_{s^{\prime}}^{\text {diff }} \cdot\left(1-\eta^{\text {diff }}\right)$ (differential source depth) $z_{b}^{\text {diff }}=2 \cdot A^{\text {diff }} \cdot D^{\text {diff }}$ (differential zero-boundary offset) $l_{\text {real }}^{\text {diff }}=\sqrt{\rho^{2}+\left(z_{a}^{\text {diff }}\right)^{2}}$ (distance from the differential source to the detector)

$l_{\text {imag }}^{\text {diff }}=\sqrt{\rho^{2}+\left(z_{a}^{\text {diff }}+2 \cdot z_{b}^{\text {diff }}\right)^{2}}$ (distance from the image of the differential source to the detector)

$\theta_{s^{\prime}}^{\text {diff }}=1-\exp \left[-\left(\frac{\mu_{s 1}^{\prime}}{\mu_{s 0}^{\prime}}\right)^{2}\right] \quad$ (scattering correction factor)

$\varepsilon_{\mathrm{eff}}^{\mathrm{diff}}=1-\left[1-\left(1-\Delta_{a}\right)^{\alpha} \cdot\left(1-\Delta_{s}^{\prime}\right)^{\beta}\right] \cdot \delta^{\gamma}$, where $(\alpha, \beta, \gamma)=$ $(100,2,1.5)$

$\mu_{\mathrm{eff}}^{\mathrm{diff}}=\sqrt{\frac{\mu_{a}^{\mathrm{diff}}}{D^{\mathrm{diff}}}} \frac{1}{1-\varepsilon_{\mathrm{eff}}^{\mathrm{diff}}} \cdot\left[1-\Delta_{a} \delta\right]^{1.5} \quad$ (differential $\quad$ effective attenuation coefficient)

$\theta_{s^{\prime}}^{\text {diff }}=1-\exp \left[-\left(\frac{\mu_{s 1}^{\prime}}{\mu_{s 0}^{\prime}}\right)^{2}\right]$ (scattering correction factor $)$

$$
\begin{aligned}
\varepsilon_{\text {strength }}^{\text {diff }}= & 1-\left[1-\left(1-\Delta_{a}\right)^{x} \cdot\left(\Delta_{s^{\prime}} \cdot \theta_{s^{\prime}}^{\text {diff }}\right)^{y}\right] \cdot \delta^{z}, \quad \text { where } \\
& (x, y, z)=(2,4,0.75) \\
\Delta_{s^{\prime}}^{\text {diff }}= & 1-\Delta_{s^{\prime}} \cdot\left[1-\exp \left(-\frac{\mu_{s 1}^{\prime}}{\mu_{s 0}^{\prime}}\right)^{2}\right](\text { a scaling factor for the } \\
& \text { differential source }) \\
S^{\text {diff }}= & S_{1}^{*} \frac{\theta_{s^{\prime}}^{\text {diff }}}{\left[1-\varepsilon_{\text {strength }}^{\text {diff }}\right)^{\text {sign }\left(\mu_{s}^{\prime}\right)}\left[1-\Delta_{a} \delta\right]^{\frac{1}{10}}}(\text { differential source strength }) .
\end{aligned}
$$

\section{Disclosures}

Daqing Piao has co-ownership interests in Surgiotonics LLC, which did not sponsor this work. The remaining authors declare no competing financial interests.

\section{Acknowledgments}

The authors are grateful to Mr. Chad Trahan and Mr. Chad Muse of LifeShare, Oklahoma City, OK, for their assistance in acquisition of the organ materials. Halen Borron's contribution to this work has been supported by a Summer Research Fellowship awarded by the Department of Pathology of the University of Oklahoma Health Sciences Center.

\section{References}

1. F. F. Jobsis, "Noninvasive, infrared monitoring of cerebral and myocardial oxygen sufficiency and circulatory parameters," Science 198(4323), 1264-1267 (1977)

2. G. Giannotti et al., "Utility of near-infrared spectroscopy in the diagnosis of lower extremity compartment syndrome," J. Trauma: Inj., Infect., Crit. Care 48(3), 396-401 (2000).

3. W. Bank et al., "Near-infrared spectroscopy in the diagnosis of mitochondrial disorders," BioFactors 7(3), 243-245 (1998).

4. C. Hock et al., "Near infrared spectroscopy in the diagnosis of Alzheimer's disease," Ann. N.Y. Acad. Sci. 777, 22-29 (1996).

5. S. Hyttel-Sorensen et al., "A phase II randomized clinical trial on cerebral near-infrared spectroscopy plus a treatment guideline versus treatment as usual for extremely preterm infants during the first three days of life (SafeBoosC): study protocol for a randomized controlled trial," Trials 14, 120 (2013).

6. R. G. Steen, K. Kitagishi, and K. Morgan, "In vivo measurement of tumor blood oxygenation by near-infrared spectroscopy: immediate effects of pentobarbital overdose or carmustine treatment," J. NeuroOncol. 22(3), 209-220 (1994).

7. A. D. Edwards et al., "Cerebral hemodynamic effects of treatment with modified natural surfactant investigated by near infrared spectroscopy," Pediatr. Res. 32(5), 532-536 (1992).

8. J. L. Zulueta et al., "Role of intraoperative regional oxygen saturation using near infrared spectroscopy in the prediction of low output syndrome after pediatric heart surgery," J. Card. Surg. 28(4), 446-452 (2013)

9. A. Demir et al., "Intraoperative 16-channel electroencephalography and bilateral near infrared spectroscopy monitorization in aortic surgery," Turk J. Anaesthesiol. Reanim. 43(4), 274-278 (2015).

10. B. Ruf et al., "Intraoperative renal near-infrared spectroscopy indicates developing acute kidney injury in infants undergoing cardiac surgery with cardiopulmonary bypass: a case-control study," Crit. Care 19, 27 (2015).

11. K. M. Gist et al., "A decline in intraoperative renal near-infrared spectroscopy is associated with adverse outcomes in children following cardiac surgery," Pediatr. Crit. Care Med. 17(4), 342-349 (2016).

12. E. L. Simmons, "Diffuse reflectance spectroscopy: a comparison of the theories," Appl. Opt. 14(6), 1380-1386 (1975).

13. G. Nogueira-Filho et al., "On site noninvasive assessment of periimplant inflammation by optical spectroscopy," J. Periodontal. Res. 46(3), 382-388 (2011).

14. S. Merritt et al., "Coregistration of diffuse optical spectroscopy and magnetic resonance imaging in a rat tumor model," Appl. Opt. 42(16), 2951-2959 (2003). 
15. S. Fantini, "A new hemodynamic model shows that temporal perturbations of cerebral blood flow and metabolic rate of oxygen cannot be measured individually using functional near-infrared spectroscopy," Physiol. Meas. 35(1), N1-N9 (2014).

16. J. Agustsson et al., "Non-contact assessment of COD and turbidity concentrations in water using diffuse reflectance UV-vis spectroscopy," Environ. Sci. Process. Impacts 16(8), 1897-1902 (2014).

17. M. P. Freitas et al., "Prediction of drug dissolution profiles from tablets using NIR diffuse reflectance spectroscopy: a rapid and nondestructive method," J. Pharm. Biomed. Anal. 39(1-2), 17-21 (2005).

18. M. Donoso and E. S. Ghaly, "Prediction of drug dissolution from tablets using near-infrared diffuse reflectance spectroscopy as a nondestructive method," Pharm. Dev. Technol. 9(3), 247-263 (2004).

19. G. A. de Oliveira et al., "Comparison of NIRS approach for prediction of internal quality traits in three fruit species," Food Chem. 143, 223230 (2014).

20. A. Rizzolo et al., "Electronic nose to detect volatile compound profile and quality changes in 'spring Belle' peach (Prunus persica L.) during cold storage in relation to fruit optical properties measured by timeresolved reflectance spectroscopy," J. Agric. Food Chem. 61(8), 1671-1685 (2013).

21. J. H. Nilsson et al., "Diffuse reflectance spectroscopy for surface measurement of liver pathology," Eur. Surg. Res. 58(1-2), 40-50 (2017).

22. U. O. Goel et al., "Feasibility of quantitative diffuse reflectance spectroscopy for targeted measurement of renal ischemia during laparoscopic partial nephrectomy," J. Biomed. Opt. 19(10), 107001 (2014).

23. D. S. Stewart, "Investigation of the optical properties of the lenses spontaneously regenerated after removal of the contents of the lens capsule of the eyes of adult rabbits," Trans. Ophthalmol. Soc. U. K. 85, 655-660 (1965).

24. Y. He et al., "Toward surface quantification of liver fibrosis progression," J. Biomed. Opt. 15(5), 056007 (2010).

25. M. L. Onozato et al., "Optical coherence tomography of human kidney," J. Urol. 183(5), 2090-2094 (2010).

26. G. Mantis and G. Zonios, "Simple two-layer reflectance model for biological tissue applications," Appl. Opt. 48(18), 3490-3496 (2009).

27. L. Pilon et al., "Simple and accurate expressions for diffuse reflectance of semi-infinite and two-layer absorbing and scattering media: erratum," Appl. Opt. 54(19), 6116-6117 (2015).

28. R. Re et al., "Effect of a thin superficial layer on the estimate of hemodynamic changes in a two-layer medium by time domain NIRS," Biomed. Opt. Express 7(2), 264-278 (2016).

29. M. Sharma et al., "Verification of a two-layer inverse Monte Carlo absorption model using multiple source-detector separation diffuse reflectance spectroscopy," Biomed. Opt. Express 5(1), 40-53 (2014).

30. D. Yudovsky and L. Pilon, "Simple and accurate expressions for diffuse reflectance of semi-infinite and two-layer absorbing and scattering media," Appl. Opt. 48(35), 6670-6683 (2009).

31. G. Zonios and A. Dimou, "Simple two-layer reflectance model for biological tissue applications: lower absorbing layer," Appl. Opt. 49(27), 5026-5031 (2010).

32. L. Zucchelli et al., "Method for the discrimination of superficial and deep absorption variations by time domain fNIRS," Biomed. Opt. Express 4(12), 2893-2910 (2013).

33. D. Q. Piao and S. Patel, "Simple empirical master-slave dual-source configuration within the diffusion approximation enhances modeling of spatially resolved diffuse reflectance at short-path and with low scattering from a semi-infinite homogeneous medium," Appl. Opt. 56(5), 1447-1452 (2017).

34. S. L. Jacques, "Optical properties of biological tissues: a review," Phys. Med. Biol. 58(11), R37-R61 (2013).

35. A. Zhang et al., "Photon diffusion in a homogeneous medium bounded externally or internally by an infinitely long circular cylindrical applicator. I. Steady-state theory," J. Opt. Soc. Am. A Opt. Image Sci. Vis 27(3), 648-662 (2010).

36. R. C. Haskell et al., "Boundary conditions for the diffusion equation in radiative transfer," J. Opt. Soc. Am. A 11(10), 2727-2741 (1994).

37. D. Piao et al., "On the geometry dependence of differential pathlength factor for near-infrared spectroscopy. I. Steady-state with homogeneous medium," J. Biomed. Opt. 20(10), 105005 (2015).
38. A. N. Bashkatov et al., "Estimation of wavelength dependence of refractive index of collagen fibers of scleral tissue," Proc. SPIE 4162, 265268 (2000).

39. Q. Wang, K. Shastri, and T. J. Pfefer, "Experimental and theoretical evaluation of a fiber-optic approach for optical property measurement in layered epithelial tissue," Appl. Opt. 49, 5309-5320 (2010).

40. Q. Wang et al., "Broadband ultraviolet-visible optical property measurement in layered turbid media," Biomed. Opt. Express 3, 1226-1240 (2012).

41. G. J. Tearney et al., "Determination of the refractive index of highly scattering human tissue by optical coherence tomography," Opt. Lett. 20(21), 2258 (1995).

42. Q. Liu and N. Ramanujam, "Scaling method for fast Monte Carlo simulation of diffuse reflectance spectra from multilayered turbid media," J. Opt. Soc. Am. A 24(4), 1011-1025 (2007).

43. J. M. Schmitt et al., "Multilayer model of photon diffusion in skin," J. Opt. Soc. Am. A 7(11), 2141-2153 (1990).

44. T. J. Farrell, M. S. Patterson, and M. Essenpreis, "Influence of layered tissue architecture on estimates of tissue optical properties obtained from spatially resolved diffuse reflectometry," Appl. Opt. 37, 19581972 (1998).

45. R. J. Hunter et al., "Haemoglobin oxygenation of a two-layer tissuesimulating phantom from time-resolved reflectance: effect of top layer thickness," Phys. Med. Biol. 47, 193-208 (2002).

46. I. Feder, H. Duadi, and D. Fixler, "Experimental system for measuring the full scattering profile of circular phantoms," Biomed. Opt. Express 6(8), 2877-2886 (2015).

Daqing Piao received his $\mathrm{PhD}$ and MS degrees in biomedical engineering from the University of Connecticut in 2003 and 2001 and his BS in physics from Tsinghua University in 1990. He is a professor in the School of Electrical and Computer Engineering (ECE) at Oklahoma State University (OSU). He has been on the faculty of ECE of OSU since 2005. His group researches biophotonics technologies to improve the outcomes of surgery, with specific interest on pretransplant donor liver assessment.

Halen Borron is a medical student trained at the College of Medicine, University of Oklahoma Health Sciences Center (OUHSC). She was the recipient of the Steven D. Chernausek Award in the 2017 Annual Harold Hamm Diabetes Center Research Symposium in OUHSC for her work of using diffuse optical spectroscopy to nondestructively assess donor organ parenchymal pathology.

Alan Hawxby received medical degree from the University of Oklahoma, followed by surgery residency at the University of Missouri-Kansas City, and a transplant surgery fellowship at Johns Hopkins Hospital, Baltimore. Currently, he is a clinical associate professor and surgical director of adult and pediatric transplantation at the University of Oklahoma. He is a liver, kidney, and pancreas transplant surgeon.

Harlan Wright is the medical director of the liver transplant program at the Oklahoma Transplant Center of OUHSC. He received his medical degree from Universidad Central de Venezuela, completing his postgraduate gastroenterology training at the University Hospital of Caracas. He spent many years at the University of Pittsburgh Transplant Center and is published in many scientific journals. He treats patients with liver disease and patients who have undergone liver transplants.

Erin M. Rubin holds James Park Dewar, MD, Professorship of Pathology in the Department of Pathology of OUHSC. She is a Gl/ hepatopancreaticobiliary and transplant pathologist. Her daily clinical responsibilities involve the diagnostic interpretation and analysis of gross and microscopic pathology material. She serves on the admission committee at the medical school of OUHSC. She is also a course director and associate course director for medical students within the department. 